\title{
Computational Fluid Dynamics Using the Adaptive Wavelet-Collocation Method
}

\author{
Yash Mehta ${ }^{1,+}\left(\mathbb{D}\right.$, Ari Nejadmalayeri ${ }^{2,+}$ and Jonathan David Regele ${ }^{3, *,+} \mathbb{C}$ \\ 1 T-3, Los Alamos National Laboratory, P.O. Box 1663, Los Alamos, NM 87545, USA; yashmehta@lanl.gov \\ 2 Wellian ${ }^{\mathrm{TM}}$ Inc., 2060 Broadway Street, Suite B-1, Boulder, CO 80302, USA; ari.nejadmalayeri@gmail.com \\ 3 XCP-4, Los Alamos National Laboratory, P.O. Box 1663, Los Alamos, NM 87545, USA \\ * Correspondence: jregele@lanl.gov \\ + These authors contributed equally to this work.
}

Citation: Mehta, Y.; Nejadmalayeri, A.; Regele, J.D. Computational Fluid Dynamics Using the Adaptive Wavelet-Collocation Method. Fluids 2021, 6, 377. https://doi.org/ 10.3390/fluids6110377

Academic Editor: Laura A. Miller

Received: 1 September 2021

Accepted: 16 October 2021

Published: 22 October 2021

Publisher's Note: MDPI stays neutral with regard to jurisdictional claims in published maps and institutional affiliations.

Copyright: (C) 2021 by the authors. Licensee MDPI, Basel, Switzerland. This article is an open access article distributed under the terms and conditions of the Creative Commons Attribution (CC BY) license (https:// creativecommons.org/licenses/by/ $4.0 /)$.

\begin{abstract}
Advancements to the adaptive wavelet-collocation method over the last decade have opened up a number of new possible areas for active research. Volume penalization techniques allow complex immersed boundary conditions to be used with high efficiency for both internal and external flows. Anisotropic methods make it possible to use body-fitted meshes while still taking advantage of the dynamic adaptability properties wavelet-based methods provide. The parallelization of the approach has made it possible to perform large high-resolution simulations of detonation initiation and fluid instabilities to uncover new physical insights that would otherwise be difficult to discover. Other developments include space-time adaptive methods and nonreflecting boundary conditions. This article summarizes the work performed using the adaptive waveletcollocation method developed by Vasilyev and coworkers over the past decade.
\end{abstract}

Keywords: wavelet-collocation; computational fluid dynamics; volume penalization; fluid instabilities; parallel algorithm; turbulence modeling; turbulence simulation; ocean modeling; detonation initiation; hot spot

\section{Introduction}

In 2010, Schneider and Vasilyev [1] documented the conception of the Adaptive Wavelet-Collocation Method in the late 1990s and its early developments in the 2000s. Over the last decade, a number of new developments have been made to the Adaptive Wavelet-Collocation Method (AWCM) that take it in new directions and generalize its application space. This review article focuses on the developments and discoveries made with the PDE solver originally developed by Vasilyev and Goldstein [2,3] in the early 2000s. In the past decade, volume penalization techniques [4] have been developed that allow complex immersed boundaries to be placed inside of computational domains with ease. Anisotropic wavelet methods have been introduced [5] that allow the use of body-fitted meshes while still using the dynamic adaptability of grid points provided using the wavelet transform. A stabilized conservative level set method has been developed [6] that improves the normal vector behavior away from an interface. Other extensions include space-time adaptive developments [7] and nonreflecting boundary conditions [8]. By far, the most impactful change has been the parallelization of the method where Zoltan dynamic load balancing $[9,10]$ is used to ensure that each processor uses nearly equal amounts of compute time [11].

The parallel implementation of the original Adaptive Wavelet Collocation Method [2] was developed in [11]. In this work, multiple approaches were investigated in order to perform an asynchronous parallel wavelet transform, i.e., with only one synchronization of the buffer-zone. They found that the most efficient solution is to skip the update stage of the wavelet transform over the entire computational domain. Hence, the Parallel AWCM (PAWCM) is a parallel extension of the AWCM with no update stage. They showed that 
skipping the update stage of wavelet transform does not change the convergence properties of the AWCM becaue the order of polynomial interpolation and accuracy of AWCM is controlled by the predict stage only. However, the major drawback of excluding the update stage of wavelet transform is the loss of zero-mean properties of the interpolating wavelet. In the PAWCM, dynamic load balancing is implemented utilizing the Zoltan library [9,10] (Figure 1). Based on the additional operations performed in the bufferzone required for asynchronous parallel wavelet transform, a theoretical limit of parallel efficiency was presented. In this theoretical efficiency, the wall-time associated with parallel communication was not taken into account.
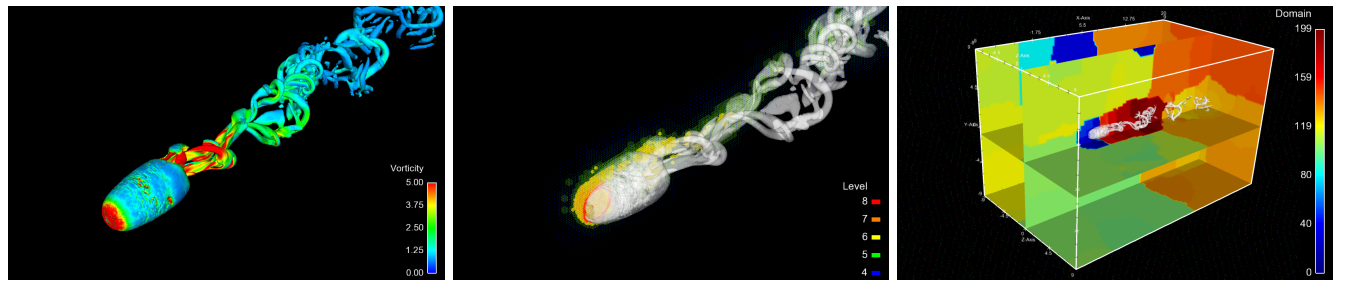

Figure 1. Wavelet-based Adaptive Direct Numerical Simulation (WA-DNS) of compressible flow past a sphere at $\operatorname{Re}=1000$ and $M a=0.7$ at effective non-adaptive resolution of $3713 \times 2305 \times 2305$. Main vortical structures, on the left. Wavelet collocation points at higher levels of resolution $(4 \leq j \leq 8)$ superimposed by iso-surfaces of main vortical structures, in the middle. Domain partitioning on 200 processes superimposed by iso-surfaces of main vortical structures, on the right. Reproduced with permission from [11], published by Elsevier 2015.

The strong scalability study of the PAWCM was presented for Coherent Vortex Simulations of homogeneous turbulence with linear forcing at a Taylor micro-scale Reynolds number of 320 on a dynamically adaptive computational grid at an effective resolution of $2048^{3}$ using as many as 2048 CPU cores (Figure 2). During this strong scalability study, the PAWCM performed at maximum theoretical efficiency up to 512 processes, while the performance for larger numbers of processes deteriorated slightly. Two main mechanisms were identified to explain the saturation of strong scalability in the PAWCM: the increase of the total number of buffer-zone points and parallel communication. The successful extension of AWCM to parallel computing has allowed various complex geometries and complex physics simulations using PAWCM since then, as outlined throughout this review article.

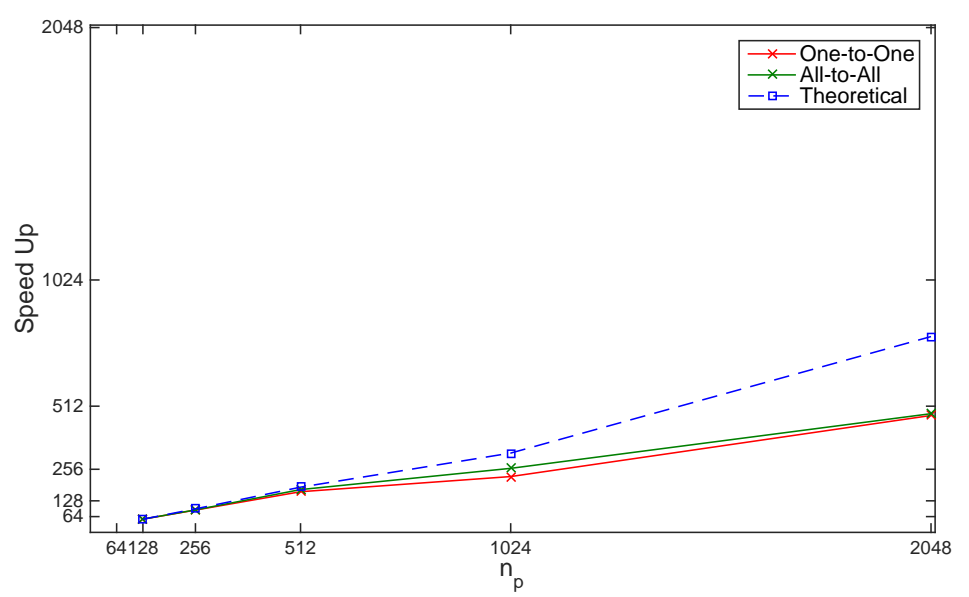

Figure 2. Theoretical and actual parallel speedup for PAWCM of Coherent Vortex Simulation (CVS) at $R e_{\lambda}=320$ on a dynamically adaptive computational grid at an effective non-adaptive resolution of $2048^{3}$. The base simulation on 128 processes is assumed to perform at theoretical efficiency. In order to evaluate the effect of interprocess communication on the parallel efficiency of PAWCM, both all-to-all and one-to-one MPI communications were implemented. Reproduced with permission from [11], published by Elsevier 2015. 
The objective of this article is to summarize the work that has been done over the past decade or more since the previous review [1] was written. The intent is not to go into significant detail on any single area, but rather to highlight the different contributions so that the interested reader can seek further detail in the relevant work. The article is organized into four different sections. Section 2 discusses the advances and tools that enable the PAWCM to be used to simulate external flows. A summary of the interesting physics that have been explored using the technique is explored in Section 3. A discussion of the turbulence models developed using wavelet-based approaches is highlighted in Section 4. Finally, Section 5 summarizes the accomplishments and suggests future directions that may be readily pursued using these developments.

\section{External Flow}

Many real-world applications of fluid mechanics comprise flow over/around complex geometries involving a wide range of time and length scales, making the computational studies of these flows extremely expensive using conventional uniform grid approaches. The inherent ability of the adaptive wavelet-collocation method (AWCM) to compress data and reduce the computational cost by avoiding over-resolution lends itself to the study of these complex flows. To accurately study the fluid-structure interaction problems, it is important to prescribe the correct numerical boundary conditions on the surfaces of the obstacles immersed in the flow. There are multiple different techniques available in the literature for prescribing the boundary conditions on the obstacles. In Section 2.1, the development of the volume penalization, curvilinear body-fitted, and the level set method for imposing the boundary conditions on the obstacles using the AWCM solver are presented. The application of the AWCM for studying external flows is discussed in Section 2.2.

\subsection{Development and Implementation of Techniques to Resolve External-Flow Using the AWCM}

In this section, a brief review of the development of various techniques to resolve the external-flow over bodies using AWCM is presented.

\subsubsection{Immersed Boundary Methods}

The viability of using the Brinkman penalization method (BPM) in conjunction with the AWCM was first explored by Vasilyev and Kevlahan [3,12]. They implemented the Brinkman penalization technique to obtain a solution for viscous incompressible flow over stationary and moving cylinders. The no-slip condition, $\mathbf{u}+\mathbf{U}=\mathbf{U}_{0}$, where $\mathbf{u}$ is the velocity field, $\mathbf{U}$ is the imposed mean velocity, and $\mathbf{U}_{0}$ is the velocity of the obstacle, was imposed on the surface of the obstacle. The mask function $\chi(\mathbf{x}, t)$ is used to distinguish between the obstacle and the fluid, and the governing equations are penalized appropriately. The accuracy of the boundary condition is controlled by parameter $\eta$ independent of spatial and temporal discretization. As $\eta \longrightarrow 0$, the original Navier-Stokes equations are recovered with appropriate boundary conditions. This makes the Brinkman method easy and cheap to implement for both, stationary and moving obstacles. The adaptive wavelet-collocation solver adapts to the mask function to resolve the obstacle and the boundary layer around it. The results for a stationary [3] cylinder are presented in Figure 3.

The incompressible BPM was extended to compressible flows by Liu and Vasilyev [13] and implemented using the AWCM solver. They noted the difficulties of straightforward extension of penalizing just the momentum and energy equations. Solid obstacles were modeled as porous objects with porosity, $\phi$, and viscous permeability approaching zero. An impedance mismatch technique was proposed to avoid the mass-energy loss due to the non-physical wave reflections from the obstacle. All the governing equations, including the conservation of mass, were penalized inside the obstacle. The results for a benchmark problem of a 2D cylindrical perturbation interacting with a stationary cylinder [13] are presented in Figure 4. Multiple acoustic reflections from the surface of the cylinder are ob- 
served in Figure 4 highlighting the capabilities of the AWCM solver to resolve compressible flow over obstacles.
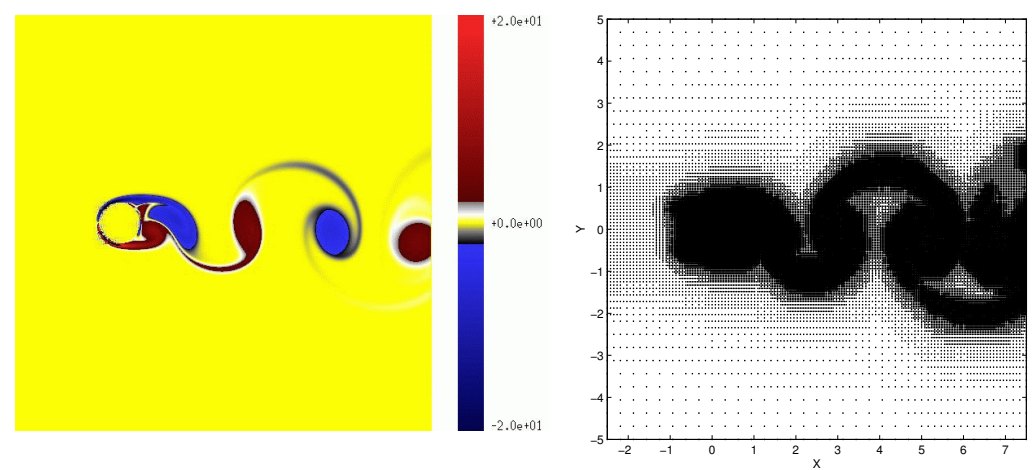

Figure 3. Viscous incompressible flow over a stationary cylinder using AWCM. The boundary conditions on the cylinder are enforced using the Brinkman penalization method. Plot of vorticity (left), grid adapting to the cylinder and the flow features (right); $t=40$. Reproduced with permission from [3], published by John Wiley \& Sons 2002.
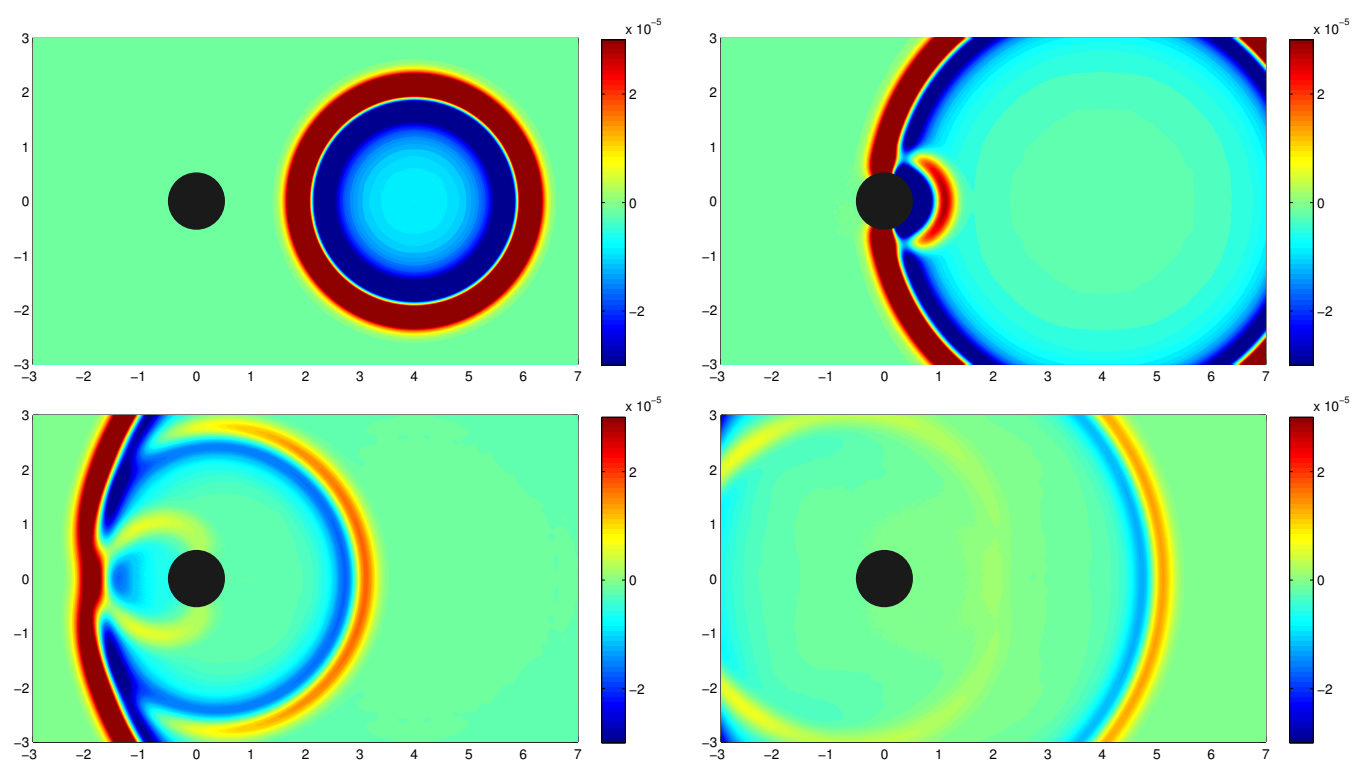

Figure 4. Contour plot of pressure for a $2 \mathrm{D}$ acoustic perturbation interacting with a stationary cylinder at time: $t=2.0$ (top left), $t=4.0$ (top right), $t=6.0$ (bottom left), and $t=8.0$ (bottom right). Reproduced with permission from [13], published by Elsevier 2007.

Brown-Dymkoski et al. [4] extended the BPM for generalized Neumann and Robin boundary conditions. The hyperbolic penalization terms with characteristics pointing inward on solid obstacles were used. The Characteristic-Based Volume Penalization (CBVP) method allows for homogeneous and inhomogeneous Dirichlet, Neumann, and Robin boundary conditions on hyperbolic and parabolic equations. Several benchmark results with heated, adiabatic, stationary, and moving cylinders using AWCM in conjunction with CBVP were demonstrated by Brown-Dymkoski et al. [4].

\subsubsection{Body-Fitted Grid on General Curvilinear Co-Ordinate Systems}

The AWCM requires the use of a rectilinear grid to efficiently use the tensorial wavelets. This can limit the application of AWCM to simple geometries. The immersed boundary methods discussed in Section 2.1.1 can be used to circumvent this limitation, but it has some drawbacks, such as approximate boundary conditions, the computational cost of resolving the non-physical flow inside the obstacle, and higher surface normal resolution 
requirements, among others. The introduction of surfaces immersed in the flow can lead to anisotropic flow features. The isotropic mesh refinement of the AWCM can over-resolve these sheet- and filament-like features. To overcome this, Brown-Dymkoski et al. [5] proposed a general framework, the Adaptive-Anisotropic Wavelet-Collocation Method (A-AWCM) to solve a system of PDEs. This new framework retains the attractive features of AWCM and allows for curvilinear anisotropic meshes, including body-fitted meshes to overcome the drawbacks of immersed boundary methods.

\subsubsection{Level Set Methods Using the AWCM Solver}

More recently, Shervani-Tabar and Vasilyev [6] implemented the stabilized conservative level set method (SCLC) using the AWCM solver. The novel SCLC method allows for volume conservation in the vicinity of the interface utilizing the compressive flux, and diffusive terms only in the normal direction of the interface. Away from the interface, the directional diffusion mechanism switches to homogeneous diffusion. Integration of the SCLC method along with the AWCM results in greater computational efficiency since the interface of the obstacle(s) is adequately resolved while allowing for low resolution away from the interface.

\subsection{Application of AWCM to Study External Flows}

S.M. Reckinger [14,15] developed immersed boundary approaches for slip and no-slip walls in ocean modeling. It was determined using asymptotic analysis that the shallow water equations combined with Brinkman penalization are hyperbolic (i.e., wave equation) in fluid regions and parabolic (i.e., diffusion equation) in continental regions. Contrary to the approach used in [13] for the Euler equations, it was determined that a boundary region also existed for the interface of the two regions that was parabolic as well. In order to ensure numerical stability in this region, a numerical boundary layer is resolved for the shallow water formulation to impose the no-slip boundary condition between the continent and ocean regions.

The accuracy of wave reflections using the immersed boundary approach for shallow water equations varies as a function of porosity and wavelength scale. The accuracy of wave reflections increases with increasing porosity until a point and, then, the accuracy decreases with increasing porosity. Since the computational cost to implement boundary conditions using this approach may be higher for conventional boundaries, the simplicity of this approach to implement complex boundaries is the primary advantage. When used in conjunction with the AWCM, it becomes a computationally efficient way to model complex boundaries. The technique was employed in demonstration simulations of sea surface heights of the North Atlantic Ocean as well as the 27 February 2010 tsunami in the Pacific Ocean (Figure 5). For each of the demonstration cases, less than $0.1 \%$ of the total available grid points were used in the simulations, illustrating the computational efficiency of the approach.

Liu et al. [13] applied the volume penalization and AWCM to perform unsteady RANS (URANS) simulations of the compressible flow around bluff bodies. 2D demonstration simulations suggested the need for a full 3D study. DNS and wavelet-based LES approaches using the volume penalization and AWCM techniques were employed by De Stefano et al. [16] to study unsteady 3D flow past a square cylinder. The results showed promise for using the combined volume penalization and AWCM techniques to obtain solutions at high Reynolds numbers and around complicated bluff bodies. De Stefano et al. [17] further extended the approach for developing a variable thresholding strategy to obtain highfidelity results in an efficient, accurate, and affordable manner for flows over bluff bodies.

The A-AWCM was leveraged by De Stefano et al. [18] to perform URANS and WAURANS simulations of external flows by incorporating a two-equation eddy-viscosity turbulence model. The body-fitted curvilinear grid was used to impose the boundary conditions directly on the discretized mesh points. The results for flow over a 2-D cylinder 
are presented in Figure 6. Section 4 has further discussion on applications of AWCM for turbulence modeling for external-flows.
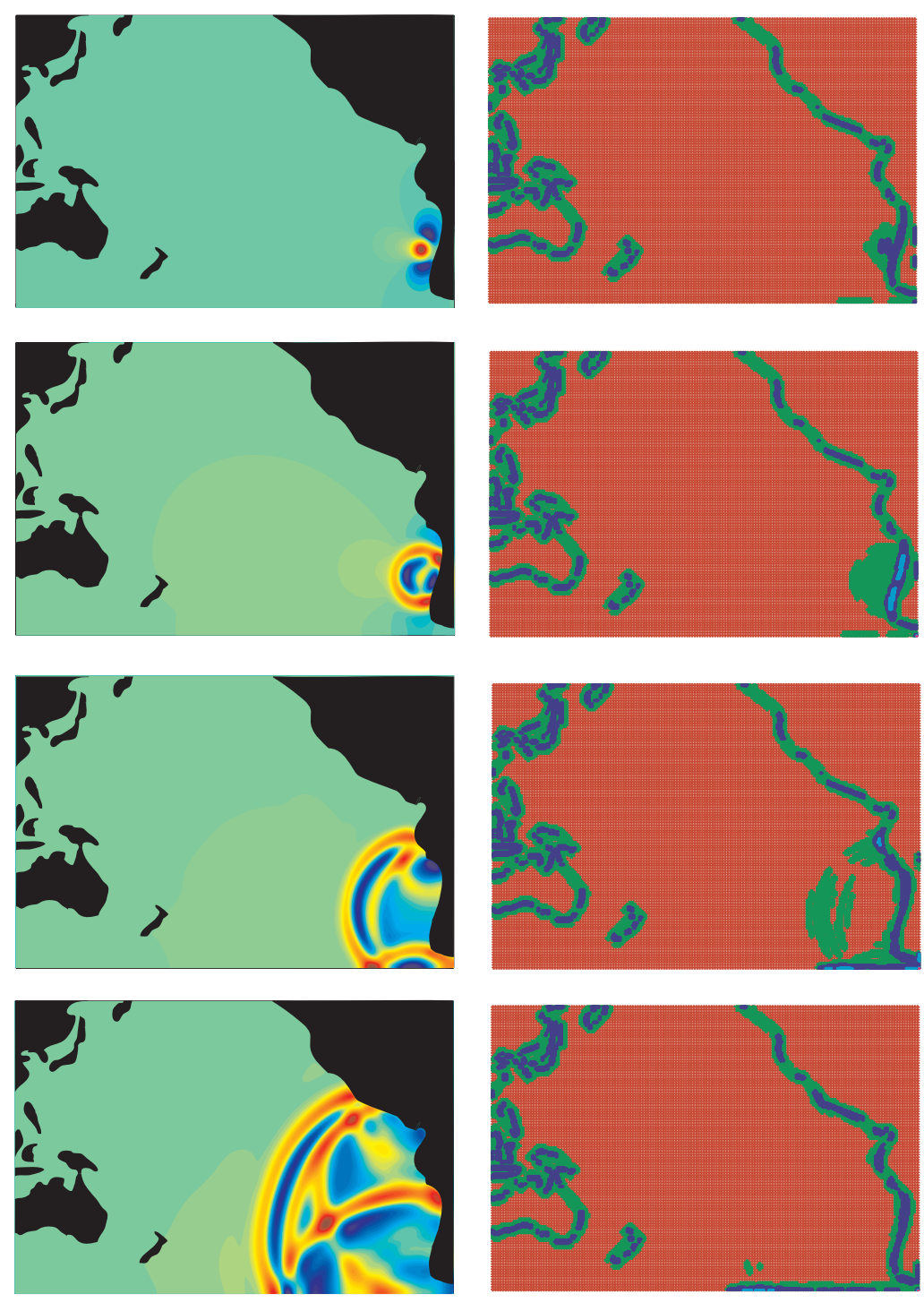

- level 5 - level 6 - level 7 - level 8 - level 9

Figure 5. Simulation results of the 27 February 2010 Pacific Ocean tsunami. Reproduced with permission from [15], published by Elsevier 2014.

The study of shock-particle interaction also suffers from the challenges associated with a large distribution of length and time scales. Numerical simulations of these problems have thus far [19-23] been performed without taking into account the effects of viscosity and/or particle motion. The AWCM solver with volume penalization and the shock-capturing schemes [24] were recently utilized by Hosseinzadeh-Nik et al. [25] to overcome these challenges and study the unsteadiness during shock-particle cloud interaction. The sources of unsteadiness were explored by quantifying the vorticity production after the passage of the shock and the difference between inviscid and viscous flow was established. 

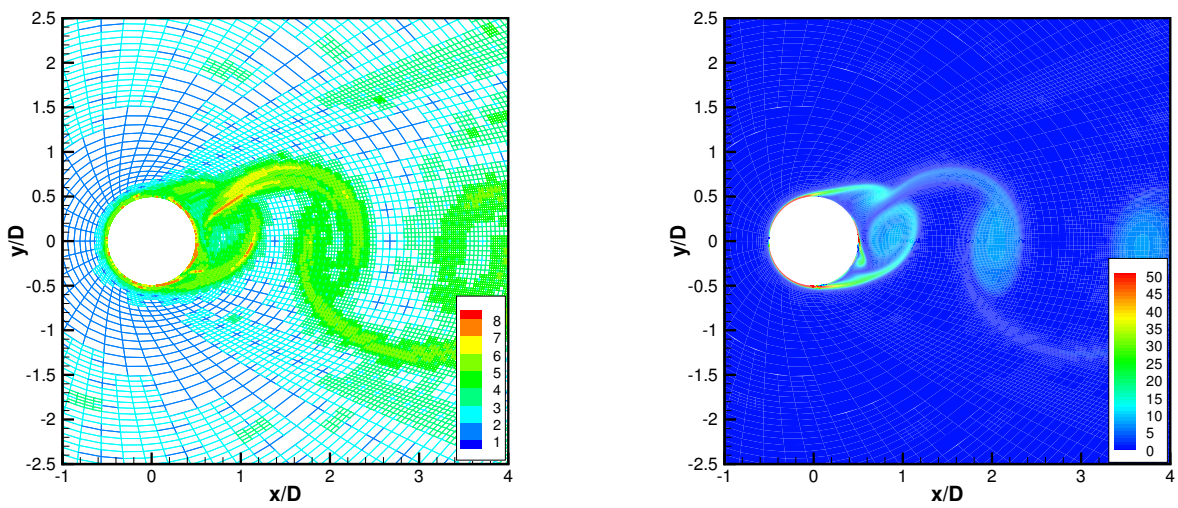

Figure 6. Results from 2D WA-URANS simulation of a cylinder. Plot of curvilinear mesh colored by adaptive levels of refinement (left), vorticity magnitude contour at a time instance during the shedding cycle (right). Reproduced with permission from [18], published by Cambridge University Press 2018.

More recently, a hard-sphere collision model was implemented by Goetsch et al. [26,27] to leverage the AWCM approach to study dense fully-resolved particle-laden flows. Each particle is modeled as a cylinder (2D) or sphere (3D) with the particle center tracked using Lagrangian particle tracking. This allows the AWCM solver to be utilized to study flows where both fluid-obstacle and obstacle-obstacle interactions are important. A demonstration of a shock-driven curtain of cylindrical particles is presented in Figure 7.

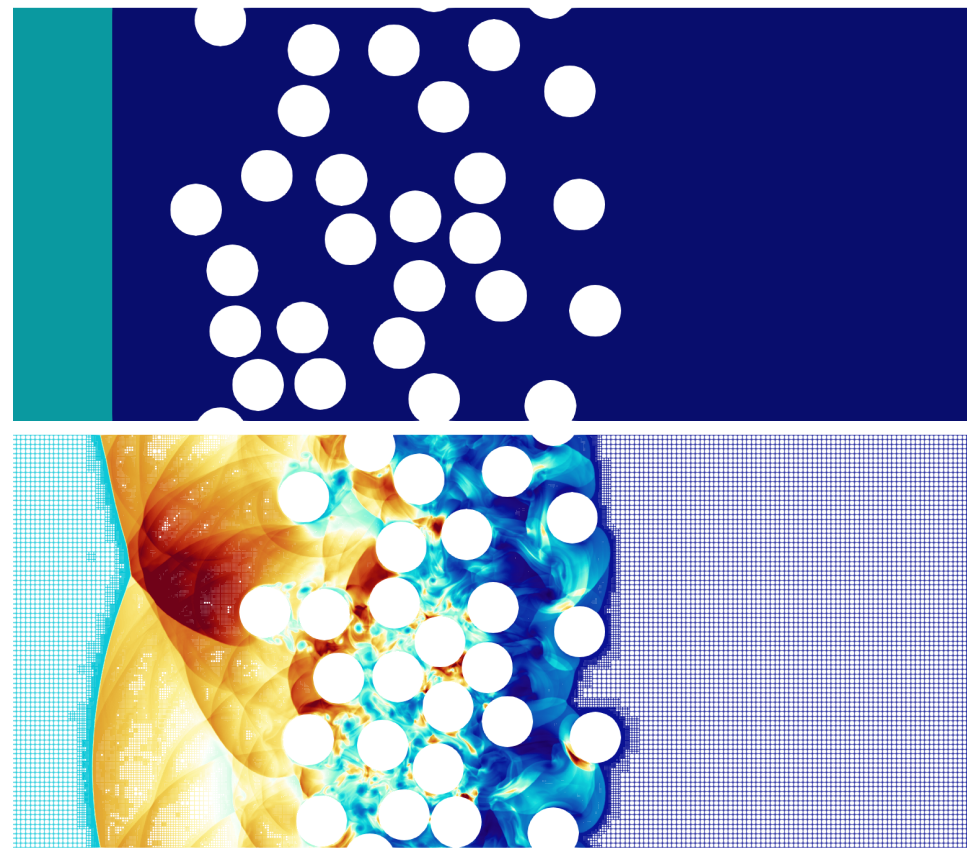

Figure 7. Cont. 


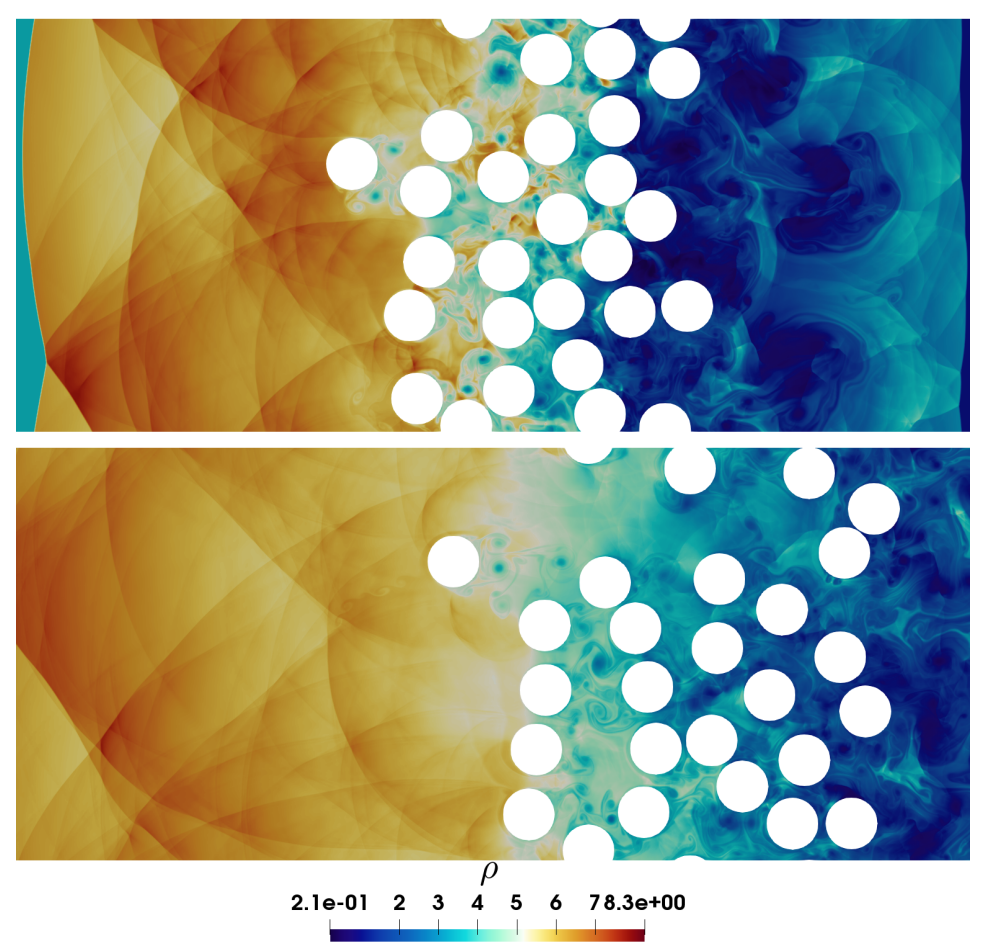

Figure 7. Contour plot of density for shock-driven curtain of particles. Reproduced from [27].

\section{Physics Exploration}

Over the past decade, the AWCM has matured to the point that it has been used to answer some fundamental scientific questions in the areas of compressible reacting flows and fluid instabilities. It has also been extended to demonstrate its ability to model heat transfer and large-scale bounded flows such as ocean modeling. The following subsections summarize these different areas.

\subsection{Detonation Initiation and Hot Spot Characterization}

Prior work by Kassoy et al. [28] showed that when power is deposited into a reactive gas over a finite time such that the fluid is inertially confined, the heat addition produces a shock wave that emanates away from the heated region that preheats the surrounding material. Once the gas is sufficiently heated and a hot spot forms with locally higher reactivity than the surrounding area, this hot spot reacts rapidly and transitions to a detonation wave. These early studies were performed using relatively low activation energies due to computational limitations involved with a multiscale problem and a uniform grid approach. In order to explore the behavior for higher activation energies, the naturally multiscale adaptive wavelet-collocation method was used.

Regele et al. [29] demonstrated using the hyperbolic approach for the AWCM [24] that the low activation energy process was pretty similar to higher activation energy reactive mixtures, but involved multiple weak hot spots to ignite and burn with little amounts of inertial confinement first. These weaker hot spots were only partially confined and further compressed the reactive media slightly. Over time, the contributions in temperature rise from each of the weak hot spots accumulated into a large enough temperature to locally form a hot spot of large enough size that the reaction is inertially confined and transitions to detonation.

Kurtz and Regele [30] extended the acoustic timescale theory to characterize the reaction of localized hot spots themselves and assess their propensity to detonate. A hot spot model comprised a temperature plateau and gradient region was proposed and analyzed for a range of excitation to acoustic timescale $\left(\tau_{e} / t_{a}\right)$ ratios. The isochoric and isobaric reaction times are fairly similar in time, so the ability of a hot spot to create compression/shock waves is mostly dependent on the size of the hot spot itself. When 
$\tau_{e} / t_{a} \gg 1$, the reaction of the plateau region commences in a nearly isochoric reaction and thereby creates a shock wave that compresses the gradient region. When $\tau_{e} / t_{a} \ll 1$, the reaction commences in an isobaric fashion and has minimal impact on the gradient region. In the case where the $\tau_{e} / t_{a}=1$, the plateau expands as it reacts and causes compression waves to propagate through the gradient region, but the heating received from this wave is less than in the inertially confined case. This work demonstrates how to classify how hot spots that form during a DDT process. The analysis was then extended [31] to multiple dimensions where the length scales in both the $x$ and $y$-directions need to be used in order to classify the hot spot ignition behavior.

In each of the studies by Kurtz and Regele [30,31], the maximum level of resolution using the PAWCM was increased until the change in peak pressure from the von Neumann spike varied by less than $1 \%$. The truly multiscale nature of the PAWCM made these simulations relatively painless to perform in both $1 \mathrm{D}$ and 2D. Although the plateau and gradient model is an improvement upon a plateau or linear gradient independently, a discontinuity still exists in the temperature gradient where the two regions join, which does not exist in natural hot spots. Reinbacher et al. [32] explored the impact of smooth temperature variation on hot spot detonability by using a superellipse to connect the temperature and temperature gradient between the plateau and linear temperature gradient regions. It was determined that hot spot detonability is highly sensitive to the rate of change of the temperature gradient for timescale ratios less than unity. In cases where the plateau/gradient model did not detonate, the smooth temperature variation from the superelliptic model often allowed a detonation to form.

Despite having obtained an improved understanding of hot spot characterization, it was still unclear how these hot spots play a role in multidimensional detonation initiation. Highly resolved simulations of 2D detonation initiation [33,34] from a thermal power deposition (Figure 8 ) demonstrated that the transition process was still pretty similar to what occurs in 1D, albeit more complex with the extra expansion and interfacial instabilities that occur in multiple dimensions. There are still multiple localized hot spots that react locally to preheat the unreacted gas until one final large hot spot reacts and transitions to a detonation. With the help of the PAWCM, the simulations were run at high enough resolution that viscous/diffusive processes implicitly present from artificial viscosity had no impact on the transition behavior. As the resolution was increased, the heat release curves in Figure 9a eventually converge demonstrating temporal resolution of the detonation initiation process. Figure $9 \mathrm{~b}$ plots the interfacial surface length, which gives an indication of the spatial structure detail present in the solution at a given time. It can be seen that for the first half of the simulation, the surface length increases in a fractal fashion with each increase in resolution due to the lack of viscosity in the simulation. Eventually, at $t \approx 12$, the spatial detail reaches a maximum and the surface length decreases until it converges approximately to the channel width of 12 at around $t=26$. This competition suggests a purely gas-dynamic multidimensional DDT process similar to what is observed in 1D exists. The interesting aspect of this work is that while highly perturbed burnt-unburnt gas interfaces are often attributed to flame acceleration and DDT, it is possible for DDT to occur without viscous/diffusive processes in play. 


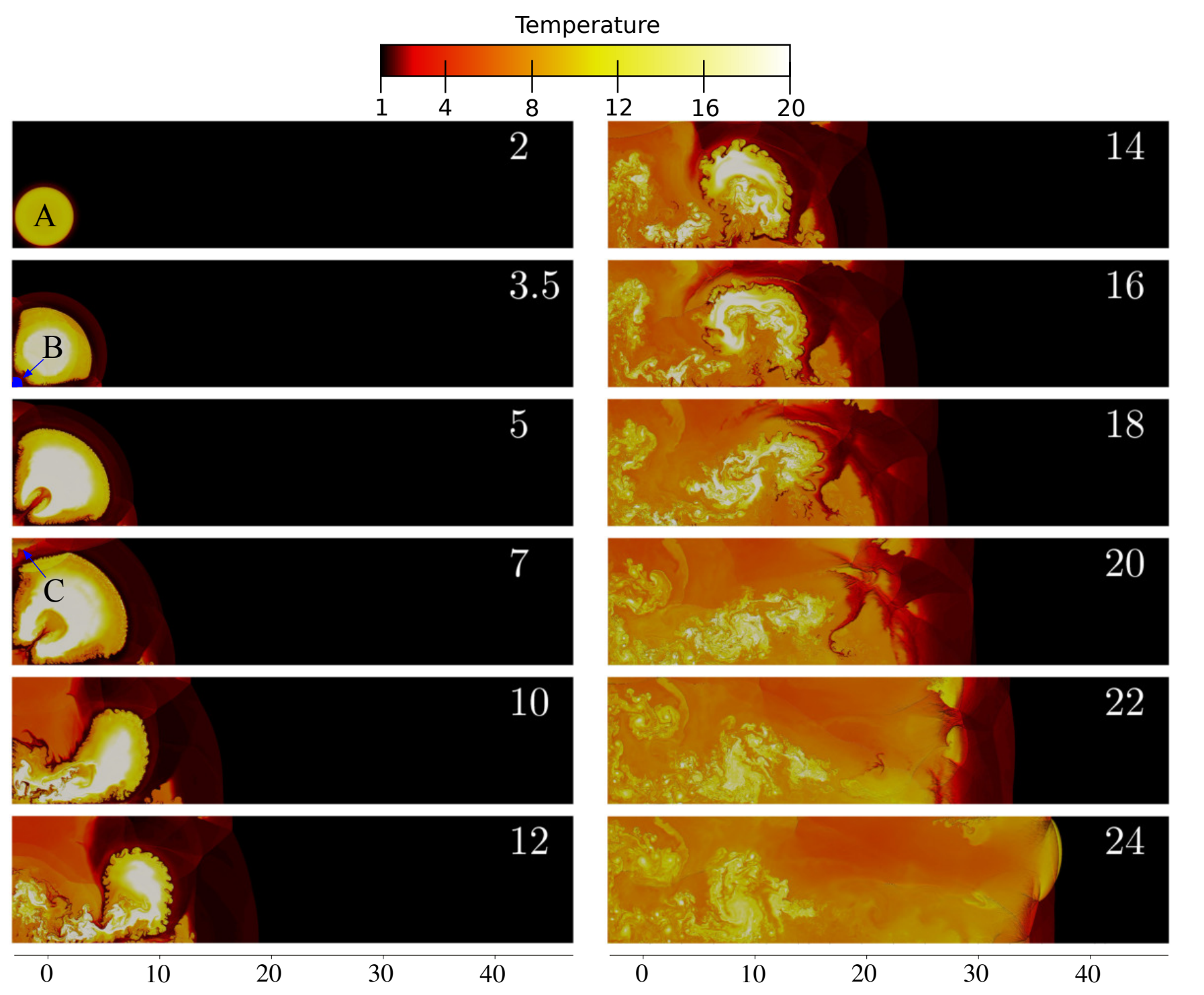

Figure 8. Sequence of temperature contours demonstrate the multidimensional detonation formation process for $j_{\max }=9$ at times $2 \leq t \leq 24$. Reproduced with permission from [34], published by Cambridge University Press 2016.

\subsection{Fluid Instabilities}

In the work described above on 2D detonation initiation, the Richtmyer-Meshkov instability is a primary source of vorticity generation at the burnt-unburnt gas interface. In most systems, the burnt-unburnt gas interface is a premixed diffusion flame that, depending on the scale of the problem, may or may not contribute significantly to the heat release and transition to detonation behavior. It can be noted that there are multiple localized explosions that emanate compression waves toward the burnt-unburnt gas interface and stretch the interface through the Richtmyer-Meshkov instability. However, most studies of this instability have focused on shock waves interacting with an interface rather than compression waves created from localized explosions where there is not only a pressure rise from an initial shock but also an expansion wave that follows and returns the gas to its original pre-compressed pressure. The reduction in computational cost to resolve the small perturbations at the burnt-unburnt gas interface was instrumental to performing simulations that were independent of numerical viscosity. 

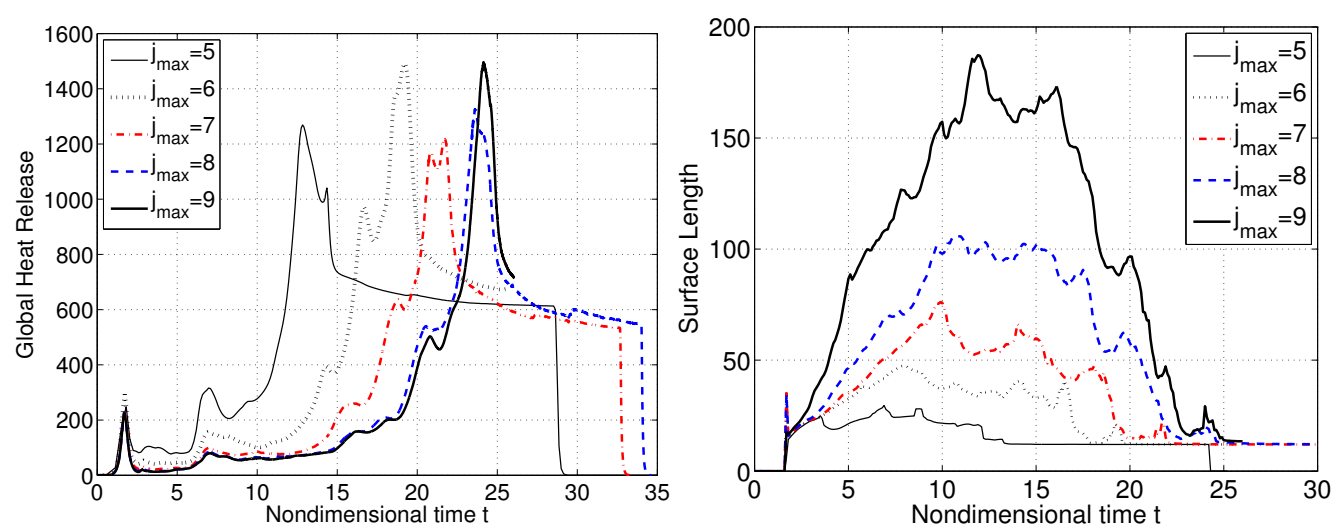

Figure 9. Global heat release profiles (left) for the 5 different levels of resolution demonstrating the differences between each successive profile diminish with increasing resolution. The burnt-unburnt gas surface length (right) demonstrates that even though the global heat release (left) shows that the detonation process is converging temporally, the spatial structures continue to increase with each increase in resolution. Reproduced with permission from [34], published by Cambridge University Press 2016.

Aslani and Regele [35] studied the impact that an expansion wave has in addition to the shock wave itself. These effects are modeled by defining a finite disturbance wave to be a shock wave followed by an expansion wave. The length of the expansion wave is varied as the ratio of the expansion fan length to the premixed laminar flame thickness $\alpha=\lambda / \delta_{f}$. Three values of $\alpha$ were used to characterize the interaction behavior such that $\alpha \in[0.1,1,10]$ and another case with $\alpha \rightarrow \infty$ indicating a shock wave.

Figure 10 shows the vorticity for each of these cases as time progresses. It can be seen that for the cases with $\alpha \leq 1$, vorticity is still created from the disturbance wave's interaction with the perturbed interface, but not to the extent of the other two cases. The $\alpha=10$ case actually stretches the interface quite a bit more than the shock-induced $(\alpha \rightarrow \infty)$ case. This occurs because the rarefaction wave interaction with the interface has additional time to produce vorticity at the interface through baroclinic torque. This causes the spike to elongate more and ultimately facilitates greater mixing.

Much of the work described above focused on fluid mixing from Richtmyer-Meshkov and Kelvin-Helmholtz instabilities. Due to the efficient grid localization properties of the AWCM, the approach is well suited to fluid instabilities in general and was also employed to analyze the Rayleigh-Taylor Instability (RTI). In [36,37], single-mode RTI was explored using the AWCM to explore the mixing of compressible and incompressible stratified fluids. Figure 11 shows a snapshot of the mole fraction, vorticity, and grid localized to regions of the high density gradient.

In compressible flows, acoustic waves may be generated at the fluid interface that may reflect from the sides of the computational domain and disturb the evolving instability interface during simulations evolved until late times. To address this issue, two different and successful boundary treatments were developed $[36,37]$ to remove acoustic disturbances from the domain. Not only do these treatments prevent acoustic waves from disturbing the interface, but they also eliminate wasting computational resources on these waves by preventing them from reentering the domain entirely. 


\section{Vorticity}

$\begin{array}{lll}-2.50 & 0.00 \quad 2.50\end{array}$

\section{$-5$}

5
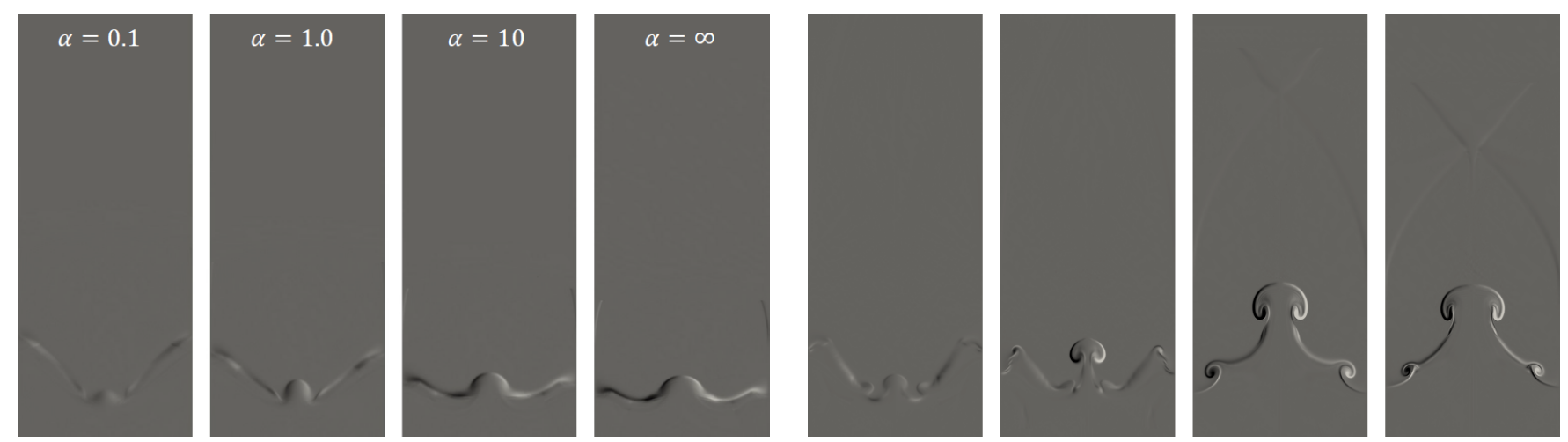

(a) $t=5$
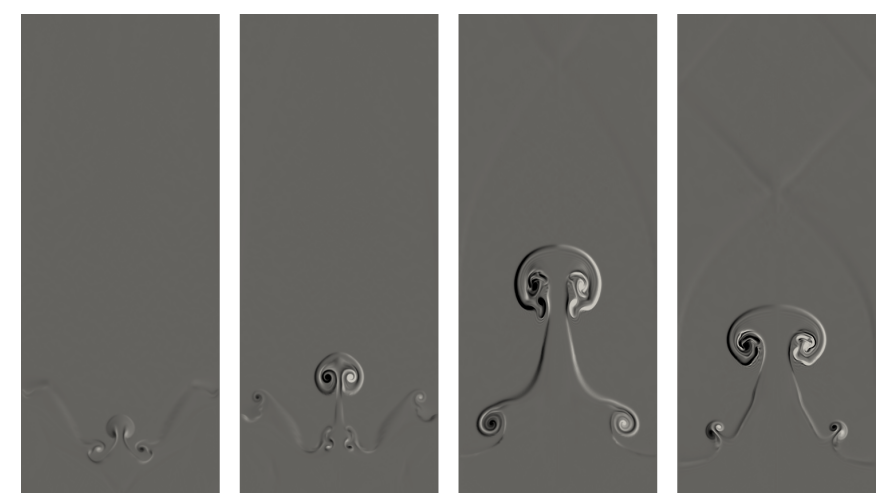

(b) $t=10$

(c) $t=15$
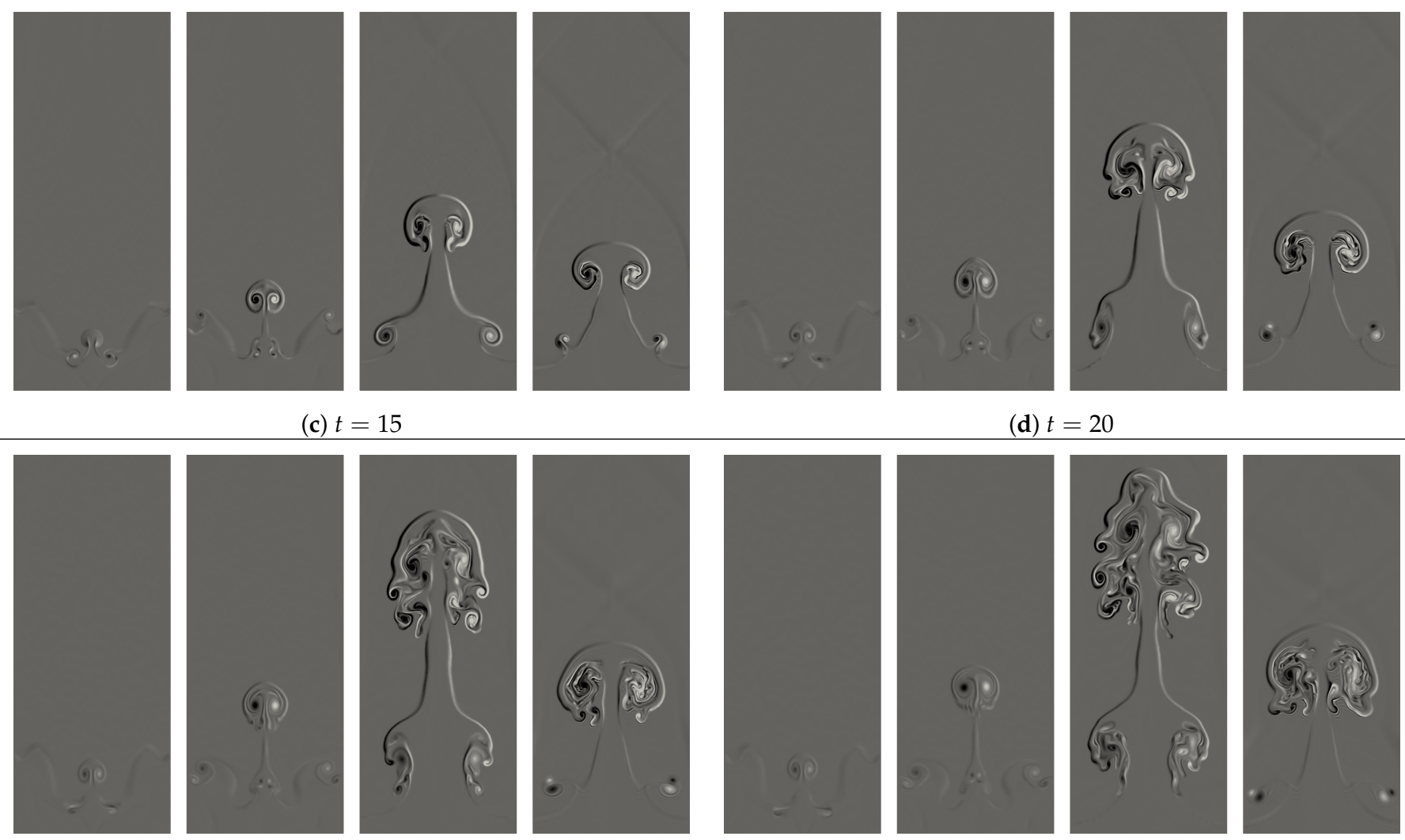

(d) $t=20$

(e) $t=25$
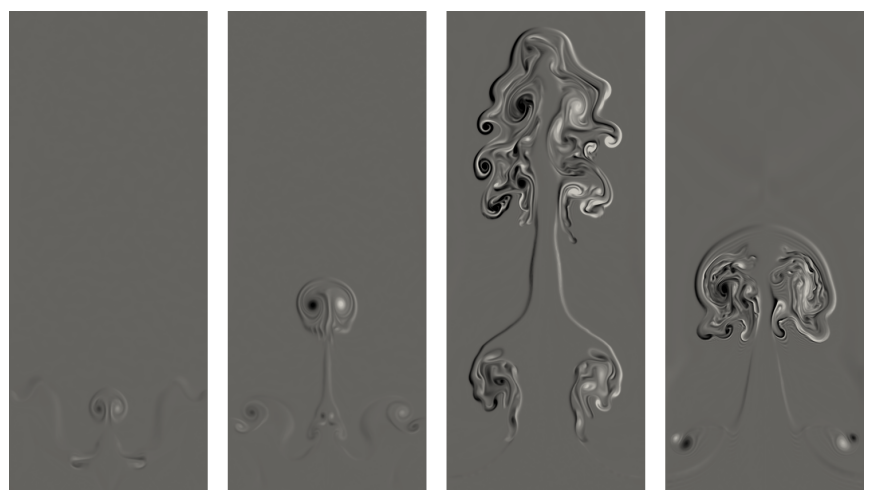

(f) $t=30$

Figure 10. Vorticity contours for different compression waves (stamped in (a)). Reproduced with permission from [35], published by Taylor and Francis 2018.

In the incompressible simulations, the results offer a unique perspective on instability growth governed by a new growth mechanism that is different from others [38]. While this mechanism may also be present in compressible cases as well, the magnitude of these effects may depend on background stratification, material compressibility, and Atwood number. It is demonstrated that this type of approach is necessary to perform high-resolution simulations of single-mode stratified RTI to understand the late time-behavior. 

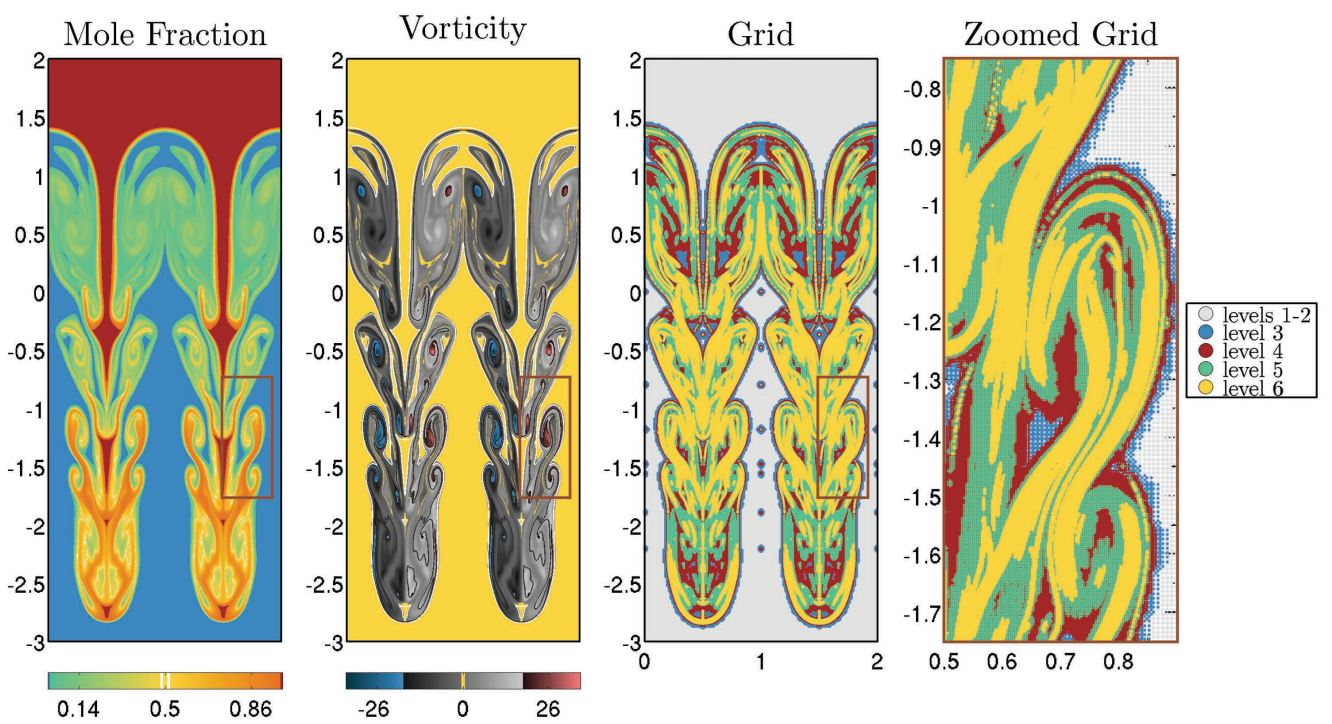

Figure 11. Instantaneous mole fraction, vorticity, and grid of Rayleigh-Taylor instability. Reproduced with permission from [37], published by Elsevier 2016.

\subsection{Heat and Mass Transfer}

In [39], the AWCM was used to solve the incompressible Navier-Stokes equations with the Boussinesq approximation to model heat transfer flows. In a variety of test problems, such as the Poisson-Boltzmann equation, shear driven, thermally driven, and heat island circulation flows, the multiresolution scheme uses a small fraction of grid cells employed by other computationally efficient methods $[40,41]$ while retaining comparable accuracy.

\subsection{Supersonic Channel Flow}

De Stefano et al. [42] integrated the different components such as A-AWCM and wavelet-based adaptive LES (WA-LES) to simulate the benchmark problem of supersonic wall-bounded turbulent flow in a channel with isothermal walls. The anisotropic minimumdissipation (AMD) approach was integrated with the A-AWCM to allow for mesh stretching in the wall-normal direction. The results demonstrated the feasibility and the effectiveness of using the novel wavelet-based adaptive method as an alternative to traditional LES for simulating the high-speed compressible wall-bounded attached turbulent regime.

\section{Turbulence Modeling}

The need for multi-resolution schemes in turbulence simulations is due to several challenges in turbulence, such as strong temporal/spatial intermittency, localized small structures in spatial/time-space, and a large range of spatial scales. Wavelet-based techniques are strong candidates due to important wavelet properties including windowed transform property (temporal/spatial localized change of scale); intrinsic adaptiveness of the waveletbased methods simply by switching on/off wavelet coefficients; signal de-noising; the existence of fast transform; coherent structures extraction (signature-recognition property); and capturing the multiscale (multi-resolution) character of turbulence. Marie Farge [43] proposed for the first time the idea of using wavelets in turbulence. The first and most prominent feature of wavelets in turbulence- a very unique wavenumber compression-is illustrated by De Stefano and Vasilyev [16], Figure 12. In addition to compression, the wavelet-threshold filtered velocity field corresponds to a wider range of wavenumbers compared with the Fourier-cutoff filtered velocity field, Figure 13. That is because of the fact that each wavelet level (scale) corresponds to a range of wavenumbers, Figure 14. This implies the second major advantage of wavelets in turbulence-the multi-resolution nature of the wavelet-based filters. The wavelet-based hierarchy of turbulence modeling is mainly categorized into wavelet-based adaptive DNS (WA-DNS), Coherent Vortex Simulation (CVS), wavelet-based adaptive LES (WA-LES) which was originally called Stochastic 
Coherent Adaptive Large Eddy Simulation (SCALES), wavelet-based adaptive Unsteady Reynolds-Averaged Navier-Stokes (WA-URANS), and wavelet-based adaptive Delayed Detached Eddv Simulation (WA-DDES).
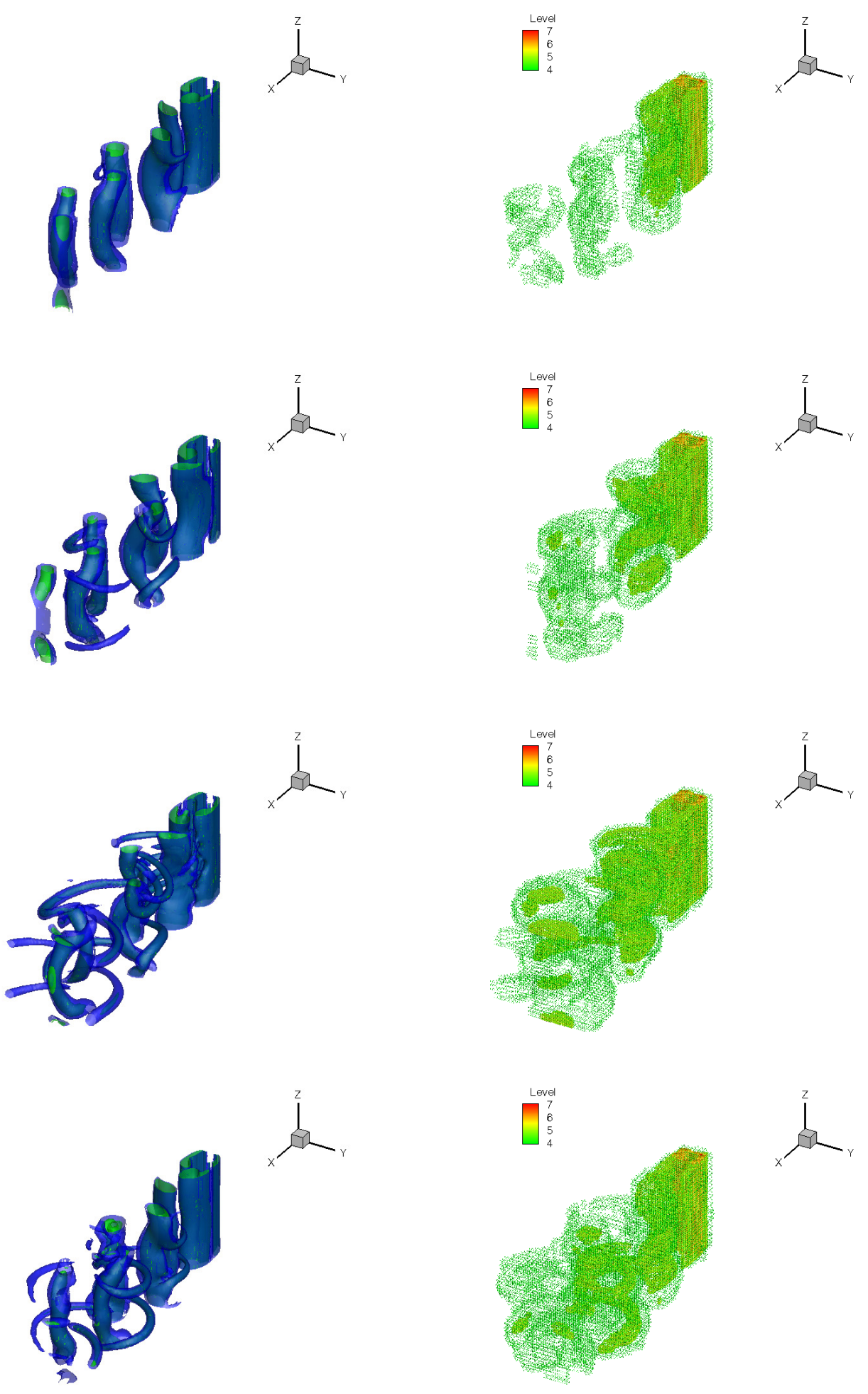

Figure 12. WA-DNS solution at $\operatorname{Re}=200$. Main vortical structures on the left. Scatter plot of the wavelet collocation points at higher levels of resolution $(4 \leq j \leq 7)$, on the right. Reproduced with permission from [16], published by Cambridge University Press 2014. 
WA-DNS [44] is an adaptive-DNS, where the wavelet-based numerical methods are used to solve the wavelet filtered Navier-Stokes equations without any model with a sufficiently small threshold level in order to ensure that the ignored-scales are not significant. The 2D spatial computational complexity for WA-DNS scales as $R e^{\frac{7}{10}}$ compared with $R e$ for non-adaptive computation [45].

CVS [46] is based on the idea that coherent modes are mostly responsible for the evolution of the turbulence as well as turbulent energy cascade and the fully developed turbulence is made of an organized coherent part; and a random incoherent part. Farge et al. [46] demonstrated that by filtering the vorticity field using orthogonal wavelet bases with an ideal threshold level, the probability density function (PDF) of the unresolved field is of the form of PDF of Gaussian white noise for homogeneous turbulence. Therefore, CVS (wavelet-filtered vorticity with an ideal threshold) is an approach to decompose the flow into deterministic and stochastic fields. Hence, in CVS, the coherent structures (the wavelet-denoised vorticity field) are simulated directly while neglecting the effect of the incoherent structures, since they provide no turbulent dissipation. CVS achieves significant compression compared with DNS. However, the number of remaining active modes are still large and the process of calculating the optimal threshold at each time-step is quite expensive since it requires the variance of the incoherent modes.

In WA-LES [47], the wavelet-filtered Navier-Stokes equations are solved using AWCM [2] which is based on the second-generation bi-orthogonal wavelet and is a compactly supported and symmetric scheme. Unlike the original formulation of CVS that is based on vorticity equations, WA-LES similar to LES solves the velocity field and it was proved that using bi-orthogonal wavelets, the PDF of the modeled portion of the SGS (wavelet-filtered out) velocity field is of the form of Gaussian white noise PDF. This fraction of the less energetic structures is a small part of the SGS field. That is to say, the velocity field is initially decomposed to more and less energetic structures by means of a wavelet threshold filter. The deterministic most energetic coherent structures are solved directly using AWCM. However, the unresolved field is not absolutely an incoherent stochastic field with no effect on the resolved field and, as a result, it needs to be modeled. Using a wavelet-threshold filter, the unresolved field is decomposed into two kinds of modes: the minority deterministic coherent SGS modes; and the majority stochastic incoherent SGS modes. The effect of these two modes on the resolved field can be modeled by deterministic SGS models and stochastic SGS models, respectively. In the original formulation of WA-LES, the extraction of the most energetic structures is achieved using a wavelet thresholding filter with an a priori prescribed threshold level.

WA-DNS, CVS, and WA-LES (formerly called SCALES) were covered in detail by the previous review paper [1] hence, herein, we continue with the recent advances.

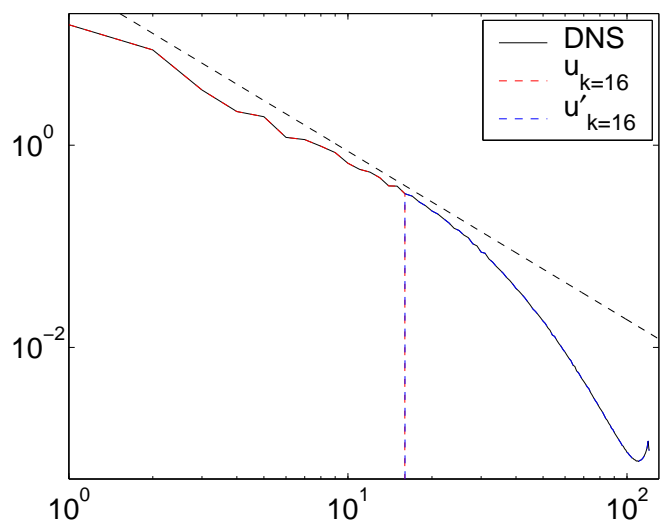

(a) Fourier Cutoff Filter

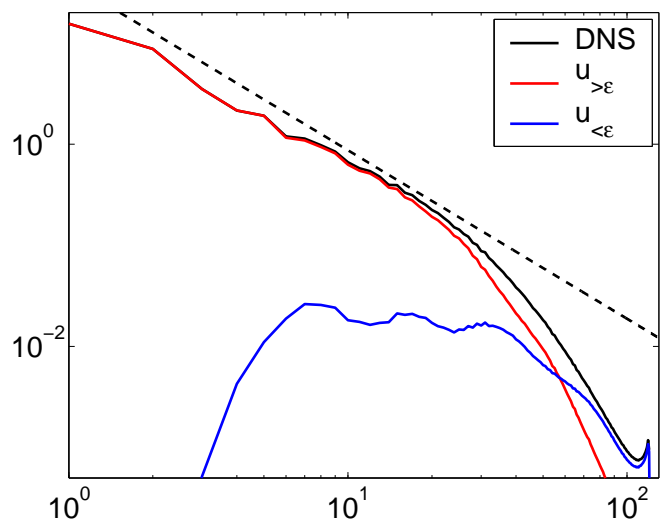

(b) Wavelet Threshold Filter

Figure 13. Filtered DNS data on $256^{3}$ grid at $R e_{\lambda} \approx 168$. Reproduced with permission from [47], published by AIP Publishing 2004. 


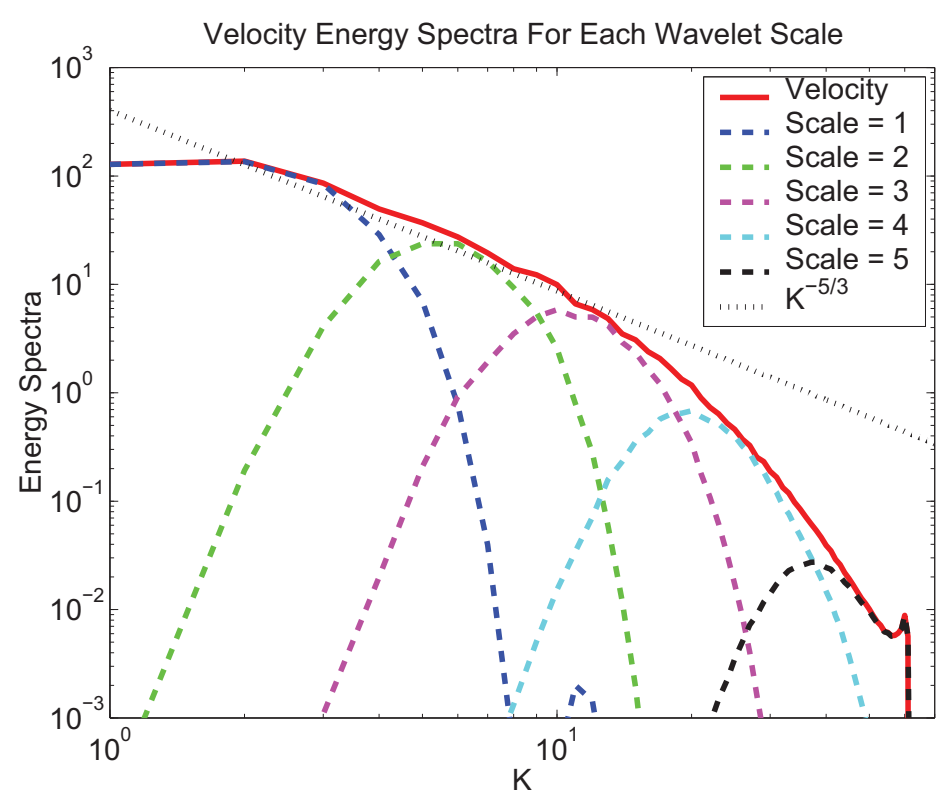

Figure 14. Velocity Energy Spectra For Each Wavelet Scale. Reproduced with permission from [47], published by AIP Publishing 2004.

\subsection{Physics-Based Adaptive Simulation-hybrid WA-DNS/CVS/WA-LES}

Analogous to the hybrid non-adaptive RANS/LES/DNS, for real physics applications, the need for hybrid WA-DNS/CVS/WA-LES is evident. Such hybrid methodologies are of great need, in particular, for wall-bounded flows and high Reynolds flows. The ability of wavelet multi-resolution analysis to detect and track the energy-containing motions that govern the dynamics of a fluid flow offers a unique hierarchical framework for modeling and simulating turbulence. The actual value of the wavelet thresholding level in waveletbased numerical simulation of turbulence directly controls the relative importance of SGS modeling as well as computational cost.

A time-varying thresholding strategy was introduced by De Stefano and Vasilyev [48], where they did not presume any subjective choice for the wavelet thresholding level. Instead, they prescribed the desired turbulence resolution, which can be expressed, for instance, in terms of the ratio of modeled to total dissipation. Such an approach completely integrates with the localized dynamic energy-based model to perform adaptive variablefidelity simulations of turbulent flows. A number of numerical experiments were conducted for both linearly forced and freely decaying homogeneous turbulence at moderate Reynolds number. For statistically unsteady turbulence, the thresholding level properly varies following the decaying nature of the flow, while maintaining the given level of turbulence resolution. They confirmed that the characteristic time scale of the readjustment of the thresholding factor is much shorter than the eddy turnover. Different WA-LES solutions with the same initial threshold were able to converge towards a certain threshold level that increases with the specified goal value for the turbulence resolution. With the increase of turbulence resolution, the SGS contribution to total dissipation decreases.

This dynamically adaptive methodology based on prescribing physically meaningful and desired criteria such as the defined turbulence resolution was further expanded to spatiotemporally varying thresholding strategy by Nejadmalayeri et al. [49]. As mentioned before, the thresholding level controls the relative importance of resolved energetic structures and residual unresolved background flow and, thus, the achieved turbulence resolution. While in [48], the wavelet thresholding filter (WTF) level automatically adapts in time to the desired turbulence resolution following the temporal evolution of the dominant flow structures, in [49], a spatiotemporally varying threshold methodology was introduced that exploits the Lagrangian representation of the variable threshold. Such a methodology can be referred to as hierarchical multi-scale adaptive variable-fidelity wavelet-based 
turbulence modeling, where the fidelity of simulations can be dynamically changed from the highest fidelity of WA-DNS all the way to the low fidelity of WA-URANS. Within this framework, only one numerical solver is utilized.

In order to consistently switch from one computational approach to another, different methods should share the same structure of governing equations. The original CVS [46] solves the wavelet-filtered vorticity equations, with the use of orthogonal Daubechies wavelets [50]. WA-LES solves wavelet-filtered Navier-Stokes equations along with an SGS model, using bi-orthogonal second-generation interpolated wavelets [51]. However, Goldstein and Vasilyev [47] showed that the turbulent velocity field can be directly decomposed into deterministic coherent and stochastic incoherent (with Gaussian probability density function) modes by applying WTF with sufficiently low values of threshold parameter, $\epsilon$. Therefore, incompressible CVS can also be based on a velocity-pressure formulation. This way, both CVS and WA-LES solve the wavelet-filtered Navier-Stokes equations, without and with SGS models, respectively, but at different thresholding levels. WA-DNS can be viewed as the solution of the same non-modeled equations with an even smaller value of the threshold parameter. In WA-LES, Goldstein et al. [52] demonstrated that the SGS dissipation increases with the WTF level and is proportional to $\epsilon^{2}$. This implies that the rate of local energy transfer from energetic resolved eddies to unresolved less energetic structures (and vice versa) can be controlled by varying $\epsilon$. A decrease of the thresholding level results in the local grid refinement with the subsequent rise of the resolved viscous dissipation, while an increase of thresholding level leads to mesh coarsening that results in the growth of the local SGS dissipation. Therefore, the WA-LES methodology can be improved by exploiting a spatially varying threshold in order to control the SGS dissipation. The idea is to vary $\epsilon$ in space wherever the level of modeled SGS dissipation deviates from a desired magnitude.

In order to vary $\epsilon$ in a physically consistent fashion, it should follow the local flow structures as they evolve in space and time. Therefore, the authors of [49] proposed a Lagrangian representation of the WTF parameter along with an artificial diffusion term in order to control the high-frequency modes in the threshold field and, consequently, the small-scale fluctuations in the velocity field due to the chaotic convective mixing. Such a Lagrangian representation of $\epsilon$ was achieved by solving an evolution equation based upon the Lagrangian path-line diffusive averaging approach [53] that uses exponentially weighted time averaging along the trajectory of a fluid particle.

This variable-fidelity methodology provided an automatic two-way transition between different wavelet-based methods within a unique framework: WA-DNS, where all significant flow structures are resolved; CVS, where coherent/incoherent flow decomposition is exploited; and WA-LES, where the energy-containing motions are resolved while modeling the effect of less energetic coherent/incoherent eddies. This dynamic variablefidelity methodology defined a new concept of turbulence model refinement, which was named $m$-refinement.

De Stefano et al. [17] expanded this spatiotemporally varying threshold to turbulent incompressible bluff-body flows. They constructed a physically meaningful forcing mechanism to take into account both laminar and turbulent regions. Their coupled waveletcollocation/volume-penalization approach with variable thresholding was utilized to solve turbulent incompressible flow around an isolated stationary prism with square crosssection at the supercritical mean flow Reynolds number of 2000, where the results are in good agreement with experimental findings and non-adaptive numerical solutions.

\subsection{WA-URANS}

As it was addressed in the previous review paper [1], unsteady RANS based on adaptive wavelet collocation method (WA-URANS) was introduced by Liu and Vasilyev [54], where they performed 2D simulations of compressible flows around a cylinder at an acoustic Reynolds number of $10^{6}$ and Mach number of 0.1632 using $k-\omega$ model. De Stefano et al. [18] extended WA-URANS to adaptive-anisotropic wavelet-collocation method (A- 
AWCM) [5] for turbulent flow past a circular cylinder at subcritical Reynolds number of 3900 using the $k-\omega$ model. Their 3D simulations demonstrated improvement in the numerical results compared with the classical URANS, which is due to the adaptive nondissipative nature of the A-AWCM method that makes it possible to reproduce relatively small scale structures. However, they found that the accurate solution of RANS equations requires the use of low wavelet threshold values compared to the ones used in WA-DNS, which is counter-intuitive when compared to the monotonically increasing threshold value for decreasing fidelity from the WA-DNS to CVS to WA-LES regimes.

Recently, Ge et al. [55] significantly improved WA-URANS by performing an extensive study for a series of both 2D and 3D simulations on various geometries including: supersonic plane channel flow, weakly compressible channel flow with periodic hill constrictions, subsonic zero-pressure-gradient flat-plate boundary-layer flow, separated flow over NASA wall-mounted hump, the Bachalo-Johnson flow (axisymmetric transonic bump flow), and flow past a circular cylinder at a subcritical Reynolds number of 3900. This work further improved the results of De Stefano et al. [18] by using a more proper far-field boundary condition, which was more in line with the ones used in conventional non-adaptive URANS. In simulations by De Stefano et al. [18], a lower than usual level of incoming turbulence intensity was used and as a result, they observed fine 3D structures for the $k-\omega$ model. Ge et al. [55] adjusted the level of incoming turbulent intensities, which increased the eddy viscosity and resulted in less pronounced 3D structures. In addition, in this comprehensive study by Ge et al. [55], both Spalart-Allmaras and $k-\omega$ models were investigated. In 3D simulations, the $k-\omega$ model was observed to be more susceptible to secondary 3D instability and to have similar trends as DNS. Furthermore, it was found that $k-\omega$ model simulations are very sensitive to the level of turbulent intensity of the incoming flow. The Spalart-Alamaras URANS model mostly resulted in 2D structures in 3D simulations.

\subsection{WA-DDES}

As shown in [18], the accurate solution of RANS equations, even on adaptive meshes as in WA-URANS, requires the use of low wavelet threshold values similar to the ones used in WA-DNS. Two distinct levels of thresholds for WA-LES and WA-URANS should be used for computations using these two different methods. The accuracy of the simulation in the WA-URANS regime is lost if an aggressively high level of $\epsilon$ in WA-LES is used. In contrast, a smaller $\epsilon$ used in WA-URANS would automatically switch WA-LES to the W-DNS regime and, thus is also unacceptable.

Ge et al. [56] developed a hybrid mathematically consistent approach in which WAURANS and WA-LES regions actively communicate with each other. This variable wavelet threshold strategy merges two distinct regions of the DDES model. The DDES model makes use of a blending function, which acts as an indicator between two regimes and switches between the RANS and LES length scales. Therefore, Ge et al. [56] proposed to use the same blending function for interpolation between the high $\epsilon_{L E S}$ and low $\epsilon_{R A N S}$ in WA-LES and WA-URANS regions. The interpolation threshold field was used to perform grid adaptation in the adaptive-anisotropic wavelet-collocation method (A-AWCM) method [5]. When turbulence contents are developed, fluctuations exist in both RANS and LES regions but the intensity of RANS fluctuations is lower. For example, in a plane channel flow, the flow structures in the RANS region near the wall are not as smooth as those in a WA-URANS simulation. Therefore, a relatively low threshold $\epsilon_{R A N S}$ would cause an unnecessary increase of grid points below the log-layer region and the expected efficiency of a hybrid-model method would be lost. In the DDES case, all variables become instantaneous and dynamically evolved turbulent eddies should be resolved. The low threshold $\epsilon_{R A N S}$ is no longer a good choice to adapt to the primary unknown variables of the evolution equations. To overcome this difficulty, a new adaptation strategy was proposed by [56]. In this novel approach, decomposition of the fields into mean and fluctuating parts results in the corresponding decomposition of the wavelet coefficients into 
mean $\bar{\epsilon}$ and fluctuating $\epsilon^{\prime}$ components, where a larger value for $\epsilon^{\prime}$ was utilized. With this decomposition, the thresholding criterion used in the original AWCM [2] is replaced by two separate criteria using mean and fluctuating components of threshold level. This new adaptation procedure provides a tighter control on the grid adaptation than the original thresholding mechanism in AWCM because:

1. It is more flexible and improves accuracy by mitigating the log-layer mismatch of the mean flow quantities with considerably smaller mean threshold $\bar{\epsilon}$ and more precise control of the relative resolution of fluctuating components using more physically relevant scales based on the turbulence intensity instead of relying on instantaneous or mean flow scales;

2. The use of a larger fluctuating threshold $\epsilon^{\prime}$ results in fewer adaptive grid points with a priori known turbulence resolution.

This new grid adaptation methodology controls the level of turbulent fluctuations independently of the mean quantities. Hence, this approach is of great interest for inhomogeneous flows, where mean quantities can have large variations. Ge et al. [56] tested this methodology for 3 cases:

1. Supersonic plane channel flow-Compared with the WA-LES, WA-DDES successfully achieved accuracy indicated by the threshold and efficiency in terms of degrees of freedom. In addition, WA-DDES resolved the typical log-layer match issue encountered in attached flows using the conventional non-adaptive DDES method;

2. Subsonic channel flow with periodic hill constrictions and massive flow separationWA-DDES was found to be in good agreement with the DNS data. Compared with WA-URANS, a great improvement was gained in terms of the separation bubble size as well as mean velocity and turbulent stress profiles. Besides, a very high compression ratio of $99.9 \%$ was achieved with the adaptive mesh size being only less than $8.0 \%$ of the non-adaptive DNS grid, while the finest allowed wavelet grid resolution was even higher than the DNS;

3. Supersonic flow over a compression ramp inducing the shock wave-turbulent boundary layer interaction-Analogous to the subsonic channel flow case, compared with WAURANS, significant improvement was gained in terms of the prediction of the shockinduced separation bubble. Compared with the reference experimental and DNS data, mean velocity and mass flux turbulence intensities were in acceptable agreement.

\subsection{Reynolds Scaling of WA-DNS, CVS, WA-LES}

Nejadmalayeri et al. [57] performed a Reynolds number scaling study of the active spatial modes for CVS and WA-LES of linearly forced homogeneous turbulence at high Reynolds numbers in a dynamic study for the first time. Their intent was to ensure that wavelet-based turbulence modeling methods can capture important flow physics of high Reynolds number flows with fewest degrees of freedom. They performed a series of linearly forced homogeneous turbulence simulations where the Reynolds number is progressively increased on a dynamically adaptive dyadic grid with effective non-adaptive resolutions of $256^{3}, 512^{3}, 1024^{3}$, and $2048^{3}$ at Taylor microscale and a Reynolds number of $R e_{\lambda}=70,120,190,320$, respectively. This study performed two different sets of simulations.

They first performed the CVS and WA-LES simulations with constant threshold levels $\epsilon=0.2,0.43$, respectively. The computational complexity with these constant threshold levels based on the $R e_{\lambda}$ scaling is compared with the DNS scaling (Figure 15). They demonstrated that the spatial modes for CVS and WA-LES scale slower than $R e_{\lambda}^{3}$ and $R e_{\lambda}^{2.75}$, respectively. These are smaller exponents compared with DNS scaling of $R e_{\lambda}^{9 / 2}$. However, in both CVS and WA-LES, the scaling deviates from the constant slope as the Reynolds number increases. Since the threshold level was kept constant, the flow is less resolved (i.e., the fidelity of the simulations decreases) as $R e_{\lambda}$ increases. 


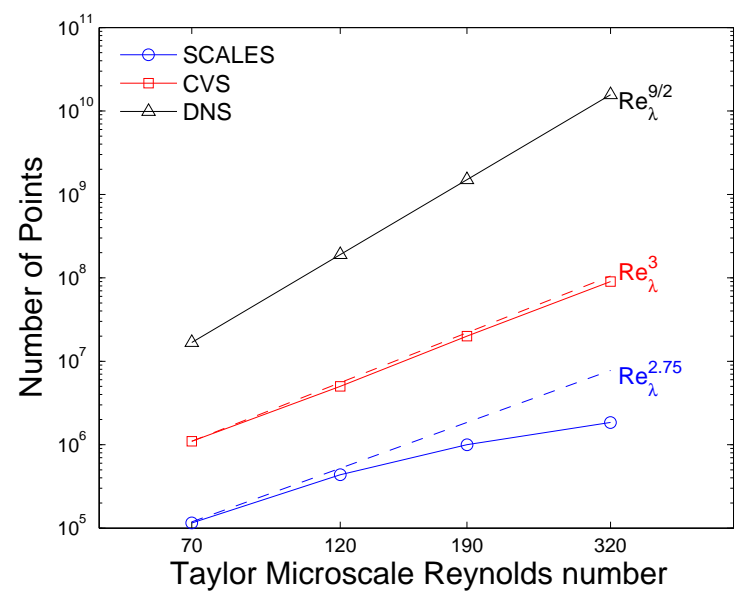

Figure 15. Reynolds number scaling of CVS and WA-LES (formerly called SCALES) with constantthreshold. Reproduced with permission from [57], published by AIP Publishing 2013.

In WA-LES, the choice of the threshold $\epsilon=0.43$ was based on the optimum threshold for $256^{3}$ simulation that is the maximum threshold at which WA-LES matches the $\mathrm{CVS}_{\epsilon_{\mathrm{opt}}}$ spectra up to inertial range [47]. By using the same threshold for larger $R e_{\lambda}$, the relative level of the resolution of the velocity field and, consequently, the kinetic energy remains constant. In CVS, a constant threshold value of $\epsilon=0.2$ corresponds to the optimal threshold value for $256^{3}$; hence, CVS using the same threshold on resolutions larger than $256^{3}$ indeed is not true CVS.

Therefore, the authors of [57] utilized variable thresholding [48,49] to maintain the percentage of resolved dissipation or the turbulence resolution approximately the same. In order to study the influence of the simulation fidelity on the Reynolds number scaling of WA-LES, another series of simulations of different turbulence resolutions was conducted. As illustrated in Figure 16 on a logarithmic scale, the slope of $R e_{\lambda}$ scaling of constantdissipation WA-LES spatial modes at least up to $1024^{3}$ remains approximately the same regardless of the level of turbulence resolution. In other words, the scaling exponent of constant-fidelity WA-LES with variable thresholding is nearly insensitive to the level of fidelity.

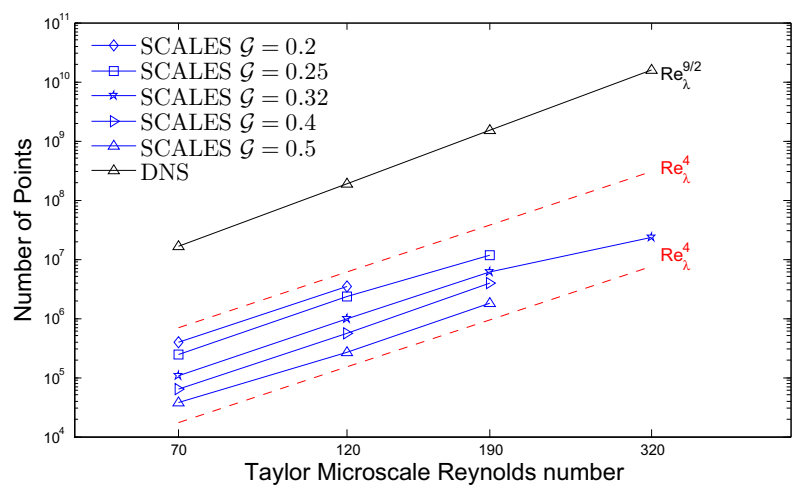

Figure 16. Reynolds scaling of constant-dissipation WA-LES (formerly called SCALES) with various goal values for the fraction of the SGS dissipation (defined as $\frac{\Pi}{\varepsilon_{\text {res }}+\Pi}$, where $\varepsilon_{\text {res }}$ is the resolved viscous dissipation and $\Pi$ is the local SGS dissipation). Reproduced with permission from [58], published by Springer Nature 2015.

This dynamic computational complexity study demonstrated that wavelet-based methods can capture flow-physics using significantly fewer degrees of freedom than both DNS and marginally resolved LES with the same level of fidelity or turbulence resolution, defined as a ratio of the subgrid-scale and the total dissipation. This study has also provided two physical conclusions as well: (1) The number of flow structures at a constant percentage 
of resolved kinetic energy-i.e., the number of energy-containing structures at a fixed level of kinetic energy - scales linearly with the Reynolds number; (2) the fractal dimensioni.e., a qualitative measure of the turbulent flow intermittency-in both constant-threshold WA-LES and CVS is close to unity, while for constant-dissipation WA-LES, it is close to two. In other words, the fractal dimension of the energy-containing structures is close to unity, while the fractal dimension of enstrophy-containing structures is close to two.

\section{Concluding Remarks}

The adaptive wavelet-collocation method developed by Vasilyev et al. has evolved considerably over the past decade. It is now possible to use the approach on parallel computer architectures up to thousands of processors. Anisotropic wavelets may be used in conjunction with body-fitted meshes while still using dynamically adaptive grid points. A level set method using wavelets has been developed that improves the normal vector behavior away from interfaces. Non-reflecting boundary conditions have been developed using volume penalization to impose complex and dynamic boundary conditions. It has even been demonstrated that full space-time adaptivity is possible. The high efficiency of the approach has been shown to be useful in ocean modeling, fluid instability growth, and particle resolved direct numerical simulations.

Recent developments in multiphase flow modeling model particles as 2D cylinders or 3D spheres using volume penalization, Lagrangian particle tracking, and hard-sphere collision models. This capability will open up new insights into how particles in multiphase flows interact with each other and enable more advanced subgrid-scale model development.

While much of the recent work has focused on toolset expansion, it has also been employed to study a range of fundamental physics questions. With its natural multiscale nature, the approach was used successfully to study acoustic timescale detonation initiation and hot pot characterization. It was shown that although more intermediate localized hot spots exist, the overall detonation initiation process of high activation energy reactive mixtures is similar to that of lower activation energies. Highly resolved simulations of purely gas-dynamic 2D detonation initiation were performed such that the viscous/diffusive contributions were negligible. Much of the detonation community focuses on the flame surface area as a way to facilitate detonation initiation. Although these processes undoubtedly help, these recent simulations show that even in a multidimensional setting, these processes are not required to form a detonation wave.

The wavelet-based hierarchy of turbulence modeling has been further expanded to WA-URANS and WA-DDES. Blending various wavelet-based methods with a series of dynamically adaptive methodologies based on prescribing physically meaningful and desired criteria has been of special attention in order to be able to model real flow problems at high Reynolds numbers. Capturing turbulent flow physics while using substantially fewer degrees of freedom than both DNS and marginally resolved LES with the same level of fidelity or turbulence resolution has been shown by a dynamic computational complexity study of linearly forced homogeneous turbulence at high Reynolds numbers. Approved for unlimited release: LA-UR-21-28667.

Funding: J.D.R. and Y.M. were funded by ASC PEM Mix and Burn.

Acknowledgments: J.D.R. and A.N. would like to thank Oleg Vasilyev for his kindness and generosity during their Ph.D. studies and beyond. He is an excellent mentor and friend.

Conflicts of Interest: The authors declare no conflict of interest.

\section{References}

1. Schneider, K.; Vasilyev, O.V. Wavelet methods in computational fluid dynamics. Annu. Rev. Fluid Mech. 2010, 42, 473-503. [CrossRef]

2. Vasilyev, O.V.; Bowman, C. Second-Generation Wavelet Collocation Method for the Solution of Partial Differential Equations. J. Comput. Phys. 2000, 165, 660-693. [CrossRef] 
3. Vasilyev, O.V.; Kevlahan, K.R. Hybrid wavelet collocation-Brinkman penalization for complex geometry flows. Int. J. Numer. Methods Fluids 2002, 40, 531-538. [CrossRef]

4. Brown-Dymkoski, E.; Kasimov, N.; Vasilyev, O.V. A characteristic based volume penalization method for general evolution problems applied to compressible viscous flows. J. Comput. Phys. 2014, 262, 344-357. [CrossRef]

5. Brown-Dymkoski, E.; Vasilyev, O.V. Adaptive-Anisotropic Wavelet Collocation Method on general curvilinear coordinate systems. J. Comput. Phys. 2017, 333, 414-426. [CrossRef]

6. Shervani-Tabar, N.; Vasilyev, O.V. Stabilized conservative level set method. J. Comput. Phys. 2018, 375, 1033-1044. [CrossRef]

7. Souopgui, I.; Wieland, S.A.; Yousuff Hussaini, M.; Vasilyev, O.V. Space-time adaptive approach to variational data assimilation using wavelets. J. Comput. Phys. 2016, 306, 253-268. [CrossRef]

8. Liu, Q.; Vasilyev, O.V. Nonreflecting boundary conditions based on nonlinear multidimensional characteristics. Int. J. Numer. Methods Fluids 2010, 62, 24-55. [CrossRef]

9. Devine, K.; Boman, E.; Heaphy, R.; Hendrickson, B.; Vaughan, C. Zoltan Data Management Services for Parallel Dynamic Applications. Comput. Sci. Eng. 2002, 4, 90-97. [CrossRef]

10. Boman, E.G.; Catalyurek, U.V.; Chevalier, C.; Devine, K.D. The Zoltan and Isorropia Parallel Toolkits for Combinatorial Scientific Computing: Partitioning, Ordering, and Coloring. Sci. Program. 2012, 20, 129-150. [CrossRef]

11. Nejadmalayeri, A.; Vezolainen, A.; Brown-Dymkoski, E.; Vasilyev, O.V. Parallel adaptive wavelet collocation method for PDEs. J. Comput. Phys. 2015, 298, 237-253. [CrossRef]

12. Kevlahan, N.K.; Vasilyev, O.V. An adaptive wavelet collocation method for fluid-structure interaction at high reynolds numbers. SIAM J. Sci. Comput. 2005, 26, 1894-1915. [CrossRef]

13. Liu, Q.; Vasilyev, O.V. A Brinkman penalization method for compressible flows in complex geometries. J. Comput. Phys. 2007, 227, 946-966. [CrossRef]

14. Reckinger, S.M.; Vasilyev, O.V.; Fox-Kemper, B. Adaptive volume penalization for ocean modeling. Ocean Dyn. 2012, 62, 1201-1215. [CrossRef]

15. Reckinger, S.M.; Vasilyev, O.V.; Fox-Kemper, B. Adaptive wavelet collocation method on the shallow water model. J. Comput. Phys. 2014, 271, 342-359. [CrossRef]

16. De Stefano, G.; Vasilyev, O.V. Wavelet-based adaptive simulations of three-dimensional flow past a square cylinder. J. Fluid Mech. 2014, 748, 433-456. [CrossRef]

17. De Stefano, G.; Nejadmalayeri, A.; Vasilyev, O.V. Wall-resolved wavelet-based adaptive large-eddy simulation of bluff-body flows with variable thresholding. J. Fluid Mech. 2016, 788, 303-336. [CrossRef]

18. De Stefano, G.; Vasilyev, O.V.; Brown-Dymkoski, E. Wavelet-based adaptive unsteady Reynolds-averaged turbulence modelling of external flows. J. Fluid Mech. 2018, 837, 765-787. [CrossRef]

19. Mehta, Y.; Jackson, T.; Zhang, J.; Balachandar, S. Numerical investigation of shock interaction with one-dimensional transverse array of particles in air. J. Appl. Phys. 2016, 119, 104901. [CrossRef]

20. Mehta, Y.; Neal, C.; Jackson, T.L.; Balachandar, S.; Thakur, S. Shock interaction with three-dimensional face centered cubic array of particles. Phys. Rev. Fluids 2016, 1, 054202. [CrossRef]

21. Mehta, Y.; Neal, C.; Salari, K.; Jackson, T.; Balachandar, S.; Thakur, S. Propagation of a strong shock over a random bed of spherical particles. J. Fluid Mech. 2018, 839, 157-197. [CrossRef]

22. Mehta, Y.; Salari, K.; Jackson, T.; Balachandar, S. Effect of Mach number and volume fraction in air-shock interacting with a bed of randomly distributed spherical particles. Phys. Rev. Fluids 2019, 4, 014303. [CrossRef]

23. Mehta, Y.; Jackson, T.; Balachandar, S. Pseudo-turbulence in inviscid simulations of shock interacting with a bed of randomly distributed particles. Shock Waves 2020, 30, 49-62. [CrossRef]

24. Regele, J.D.; Vasilyev, O.V. An Adaptive Wavelet-Collocation Method for Shock Computations. Int. J. Comput. Fluid Dyn. 2009, 23, 503-518. [CrossRef]

25. Hosseinzadeh-Nik, Z.; Subramaniam, S.; Regele, J.D. Investigation and quantification of flow unsteadiness in shock-particle cloud interaction. Int. J. Multiph. Flow 2018, 101, 186-201. [CrossRef]

26. Goetsch, R.J.; Regele, J.D. Discrete element method prediction of particle curtain properties. Chem. Eng. Sci. 2015, 137, 852-861. [CrossRef]

27. Mehta, Y.; Goetsch, R.J.; Regele, J.D. Particle resolved direct numerical simulations using the adaptive wavelet collocation method to study shock interacting with moving, colliding particles. J. Comput. Phys. 2021, under review.

28. Kassoy, D.R.; Kuehn, J.A.; Nabity, M.W.; Clarke, J.F. Detonation initiation on the microsecond time scale: DDTs. Combust. Theory Model. 2008, 12, 1009-1047. [CrossRef]

29. Regele, J.D.; Kassoy, D.R.; Vasilyev, O.V. Effects of high activation energies on acoustic timescale detonation initiation. Combust. Theory Model. 2012, 16, 650-678. [CrossRef]

30. Kurtz, M.D.; Regele, J.D. Acoustic timescale characterisation of a one-dimensional model hot spot. Combust. Theory Model. 2014, 18, 532-551. [CrossRef]

31. Kurtz, M.D.; Regele, J.D. Acoustic timescale characterization of symmetric and asymmetric multidimensional hot spots. Combust. Theory Model. 2014, 18, 711-729. [CrossRef]

32. Reinbacher, F.; Regele, J.D. Influence of smooth temperature variation on hotspot ignition. Combust. Theory Model. 2018, 22, 110-130. [CrossRef] 
33. Regele, J.D.; Kassoy, D.R.; Vezolainen, A.; Vasilyev, O.V. Indirect detonation initiation using acoustic timescale thermal power deposition. Phys. Fluids 2013, 25, 1-2. [CrossRef]

34. Regele, J.D.; Kassoy, D.R.; Aslani, M.; Vasilyev, O.V. Evolution of detonation formation initiated by a spatially distributed, transient energy source. J. Fluid Mech. 2016, 802, 305-332. [CrossRef]

35. Aslani, M.; Regele, J.D. Numerical simulation of finite disturbances interacting with laminar premixed flames. Combust. Theory Model. 2018, 22, 812-833. [CrossRef]

36. Reckinger, S.J.; Livescu, D.; Vasilyev, O.V. Adaptive wavelet collocation method simulations of Rayleigh-Taylor instability. Phys. Scr. 2010, 2010, 014064. [CrossRef]

37. Reckinger, S.J.; Livescu, D.; Vasilyev, O.V. Comprehensive numerical methodology for direct numerical simulations of compressible Rayleigh-Taylor instability. J. Comput. Phys. 2016, 313, 181-208. [CrossRef]

38. Wei, T.; Livescu, D. Late-time quadratic growth in single-mode Rayleigh-Taylor instability. Phys. Rev. E 2012, 86, 1-14. [CrossRef]

39. Alam, J.M.; Kevlahan, N.K.; Vasilyev, O.V.; Hossain, Z. A multiresolution model for the simulation of transient heat and mass transfer. Numer. Heat Transf. Part B Fundam. 2012, 61, 147-170. [CrossRef]

40. Ghia, U.; Ghia, K.N.; Shin, C.T. High-Re Solutions for Incompressible Flow Using the Navier-Stokes Equations and a Multigrid Method. J. Comput. Phys. 1982, 48, 387-411. [CrossRef]

41. Botella, O.; Peyret, R. Benchmark spectral results on the lid-driven cavity flow. Comput. Fluids 1998, 27, 421-433. [CrossRef]

42. De Stefano, G.; Brown-Dymkoski, E.; Vasilyev, O.V. Wavelet-based adaptive large-eddy simulation of supersonic channel flow. J. Fluid Mech. 2020, 901. [CrossRef]

43. Farge, M.; Rabreau, G. Transformee en ondelettes pour detecter et analyser les structures coherentes dans les ecoulements turbulents bidimensionnels. C. R. Acad. Sci. Paris 1988, II, 1479-1486.

44. Fröhlich, J.; Schneider, K. Numerical simulation of decaying turbulence in an adaptive wavelet basis. Appl. Comput. Harmon. Anal. 1996, 3, 393-397. [CrossRef]

45. Kevlahan, N.K.R.; Alam, J.; Vasilyev, O.V. Scaling of space-time modes with Reynolds number in two-dimensional turbulence. J. Fluid Mech. 2007, 570, 217-226. [CrossRef]

46. Farge, M.; Schneider, K.; Kevlahan, N. Non-Gaussianity and coherent vortex simulation for two-dimensional Nurbulence using an adaptive orthogonal wavelet basis. Phys. Fluids 1999, 11, 2187-2201. [CrossRef]

47. Goldstein, D.E.; Vasilyev, O.V. Stochastic coherent adaptive large eddy simulation method. Phys. Fluids 2004, 16, 2497-2513. [CrossRef]

48. De Stefano, G.; Vasilyev, O.V. A fully adaptive wavelet-based approach to homogeneous turbulence simulation. J. Fluid Mech 2012, 695, 149-172. [CrossRef]

49. Nejadmalayeri, A.; Vezolainen, A.; De Stefano, G.; Vasilyev, O.V. Fully adaptive turbulence simulations based on Lagrangian spatio-temporally varying wavelet thresholding. J. Fluid Mech. 2014, 749, 794-817. [CrossRef]

50. Daubechies, I. Ten Lectures on Wavelets; SIAM: Philadelphia, PA, USA, 1992.

51. Sweldens, W. The lifting scheme: A custom-design construction of biorthogonal wavelets. Appl. Comput. Harmon. Anal. 1996, 3, 186-200. [CrossRef]

52. Goldstein, D.E.; Vasilyev, O.V.; Kevlahan, N.K. CVS and SCALES simulation of 3-D isotropic turbulence. J. Turbul. 2005, 6, 1-20. [CrossRef]

53. Vasilyev, O.V.; De Stefano, G.; Goldstein, D.E.; Kevlahan, N.K. Lagrangian dynamic SGS model for stochastic coherent adaptive large eddy simulation. J. Turbul. 2008, 9, 1-14. [CrossRef]

54. Liu, Q.; Vasilyev, O.V. Hybrid adaptive wavelet collocation-Brinkman penalisation method for unsteady RANS simulations of compressible flow around bluff bodies. In Proceedings of the 36th AIAA Fluid Dynamics Conference, San Francisco, CA, USA, 5-8 June 2006; Collection of Technical Papers; Volume 1, pp. 730-739. [CrossRef]

55. Ge, X.; Vasilyev, O.V.; De Stefano, G.; Yousuff Hussaini, M. Wavelet-Based Adaptive Unsteady Reynolds-Averaged Navier-Stokes Simulations of Wall-Bounded Compressible Turbulent Flows. AIAA J. 2020, 58, 1529-1549. [CrossRef]

56. Ge, X.; Vasilyev, O.V.; Hussaini, M.Y. Wavelet-based adaptive delayed detached eddy simulations for wall-bounded compressible turbulent flows. J. Fluid Mech. 2019, 873, 1116-1157. [CrossRef]

57. Nejadmalayeri, A.; Vezolainen, A.; Vasilyev, O.V. Reynolds number scaling of coherent vortex simulation and stochastic coherent adaptive large eddy simulation. Phys. Fluids 2013, 25, 1-15. [CrossRef]

58. Nejadmalayeri, A.; Vasilyev, O.V.; Vezolainen, A. Computational Complexity of Adaptive LES with Variable Fidelity Model Refinement. In Direct and Large-Eddy Simulation IX; Fröhlich, J., Kuerten, H., Geurts, B.J., Armenio, V., Eds.; Springer International Publishing: Cham, Switzerland, 2015; pp. 149-153. 